\title{
Intramolecular Additions of Alcohols and Carboxylic Acids to Inert Olefins Catalyzed by Silver(I) Triflate
}

\author{
Cai-Guang Yang, Nicholas W. Reich, Zhangjie Shi, Chuan He* \\ Department of Chemistry, the University of Chicago, \\ 5735 S. Ellis Avenue, Chicago, IL 60617, \\ E-mail: chuanhe@uchicago.edu
}

General Methods. Catalytic cyclization reactions were performed in sealed oven-dried glass tubes under an atmosphere of dry nitrogen. Flash column chromatography was performed employing EM Science Geduran silica gel $60(35-75 \mu \mathrm{m})$, and eluting with npentane/ethyl ether. Thin layer chromatography was performed on EM Science silica gel 60 F254 plates $(250 \mu \mathrm{m})$. NMR spectra were recorded on operating at Bruker DRX$400 / 500$ spectrometer for ${ }^{1} \mathrm{H} \mathrm{NMR}$ and $100 / 125 \mathrm{MHz}$ for ${ }^{13} \mathrm{C} \mathrm{NMR}$ in $\mathrm{CDCl}_{3}$ unless noted otherwise and were referenced to tetramethylsilane (TMS) at $0.00 \mathrm{ppm}$. The chemical shifts are expressed in ppm and coupling constants are given in $\mathrm{Hz}$. Data for ${ }^{1} \mathrm{H}$ NMR are recorded as follows: chemical shift (ppm), multiplicity (s, singlet; d, doublet; $t$, triplet; $\mathrm{m}$, multiplet), coupling constant $(\mathrm{Hz})$, integration. Data for ${ }^{13} \mathrm{C} \mathrm{NMR}$ are reported in terms of chemical shift $(\delta, \mathrm{ppm})$. Low-resolution mass spectra were obtained from the Mass Spectrometry Facility at the University of Chicago. Elemental analyses were performed by Microanalytical Laboratory at University of Illinois at Urbana-Champaign. Silver triflate was purchased from Aldrich and used as received. 1,2-Dichloroethane (DCE) was redistilled over $\mathrm{CaH}_{2}$ before used. THF was freshly distilled over sodium benzoquinone ketyl under dry nitrogen.

Most products are known compounds, and the references are given. For unknown products, copies of ${ }^{1} \mathrm{H}$ NMR and ${ }^{13} \mathrm{C}$ NMR spectrum are attached at the end. Elemental analysis for product (Table 2, entry 4 ) is also obtained.

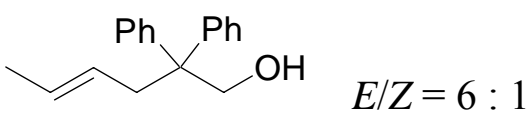

2,2-Diphenyl-4-hexen-1-ol (Table 2, entry 3). ${ }^{1}$ To a solution of methyl diphenylacetate $(2.26 \mathrm{~g}, 10.0 \mathrm{mmol})$ in THF was added a solution of LDA $(1.8 \mathrm{M}, 6.8 \mathrm{~mL}, 12.0 \mathrm{mmol})$ dropwise at $-78{ }^{\circ} \mathrm{C}$ and the resulting solution was stirred for additional $20 \mathrm{~min}$. Crotyl bromide $(1.45 \mathrm{~mL}, 12.0 \mathrm{mmol})$ was added and the reaction mixture was warmed to $\mathrm{rt}$, stirred overnight, quenched with $\mathrm{HCl}(3 \mathrm{~N}, 10.0 \mathrm{~mL})$, and extracted with ether $(3 \times 50$ $\mathrm{mL})$. The combined ether extracts were washed with brine $(40 \mathrm{~mL})$, dried $\left(\mathrm{MgSO}_{4}\right)$, and concentrated under vacuum. The residue was purified by silica gel chromatography to give methyl 2,2-dipheneyl-4-pentenoate $(2.24 \mathrm{~g}, 80 \%)$ as a viscous colorless oil.

To a suspension of $\mathrm{LiAlH}_{4}(380 \mathrm{mg}, 10.0 \mathrm{mmol})$ in THF $(10 \mathrm{~mL})$ was slowly added a solution of methyl 2,2-dipheneyl-4-pentenoate $(1.12 \mathrm{~g}, 5 \mathrm{mmol})$ at $0{ }^{\circ} \mathrm{C}$. The resulting mixture was refluxed for $2 \mathrm{~h}$, and quenched by carefully addition of water $(2.5 \mathrm{~mL})$ and aqueous $\mathrm{NaOH}(15 \%, 3 \mathrm{~mL})$ at $0{ }^{\circ} \mathrm{C}$. The resulting suspension was filtered through Celite and eluted with ether. The ether eluant was dried over $\mathrm{MgSO}_{4}$ and concentrated under vacuum. The residue was purified by silica gel chromatography to give the title compound $(0.91 \mathrm{~g}, 90 \%)$ as colorless oil. For E: ${ }^{1} \mathrm{H}$ NMR $(500 \mathrm{MHz}) \delta 7.28-7.25(\mathrm{~m}$, 
4H), $7.20-7.15(\mathrm{~m}, 6 \mathrm{H}), 5.48(\mathrm{~m}, 1 \mathrm{H}), 5.04(\mathrm{~m}, 1 \mathrm{H}), 4.09(\mathrm{~d}, J=6.5 \mathrm{~Hz}, 2 \mathrm{H}), 2.87(\mathrm{~d}, J$ $=7.0 \mathrm{~Hz}, 2 \mathrm{H}), 1.55(\mathrm{~d}, J=6.5 \mathrm{~Hz}, 3 \mathrm{H}), 1.27(\mathrm{t}, J=7.0 \mathrm{~Hz}, 1 \mathrm{H}) ;{ }^{13} \mathrm{C}$ NMR $(125 \mathrm{MHz}) \delta$ $145.5,128.6,128.2,128.1,128.0,126.6,126.2,67.9,51.7,39.7,17.9 ; \mathrm{MS}(\mathrm{m} / \mathrm{z}, \mathrm{EI}) 235$ $\left[(\mathrm{M}-\mathrm{OH})^{+}\right]$.<smiles>C=C(C)CC(CO)c1cccc2ccccc12</smiles>

2-(1-Naphenyl)-4-methyl-4-penten-1-ol (Table 2, entry 4). The title compound was prepared in $72 \%$ yield as a colorless oil from reaction of methyl 1-naphthaleneacetate and 3-bromo-2-methylpropene employing a procedure analogous to the one used to synthesize 2,2-diphenyl-4-hexen-1-ol. ${ }^{1} \mathrm{H}$ NMR $(500 \mathrm{MHz}) \delta 8.21(\mathrm{~d}, J=8.5 \mathrm{~Hz}, 1 \mathrm{H})$, $7.90(\mathrm{~d}, J=8.0 \mathrm{~Hz}, 1 \mathrm{H}), 7.77(\mathrm{~d}, J=8.0 \mathrm{~Hz}, 1 \mathrm{H}), 7.58-7.40(\mathrm{~m}, 4 \mathrm{H}), 4.02(\mathrm{~m}, 1 \mathrm{H}), 3.91$ $(\mathrm{t}, J=5.5 \mathrm{~Hz}, 2 \mathrm{H}), 2.68(\mathrm{dd}, J=14.5$ and $7.5 \mathrm{~Hz}, 1 \mathrm{H}), 2.56(\mathrm{dd}, J=14.5$ and $7.0 \mathrm{~Hz}$, $1 \mathrm{H}), 1.79(\mathrm{~s}, 3 \mathrm{H}), 1.65(\mathrm{t}, J=5.5 \mathrm{~Hz}, 1 \mathrm{H}) ;{ }^{13} \mathrm{C} \mathrm{NMR}(125 \mathrm{MHz}) \delta 143.7,138.1,134.1$, $132.2,129.0,127.1,126.0,125.5,125.4,123.8,122.8,112.4,66.7,40.7,39.7,22.5$; MS $(\mathrm{m} / \mathrm{z}, \mathrm{EI}) 226\left(\mathrm{M}^{+}\right)$.<smiles>C=CC(CCO)Pc1ccccc1</smiles>

3-Phenyl-4-penten-1-ol (Table 2, entry 5). The title compound was synthesized according to a published procedure. ${ }^{2}{ }^{1} \mathrm{H}$ NMR $(400 \mathrm{MHz}) \delta 7.34-7.30(\mathrm{~m}, 2 \mathrm{H}), 7.23-$ $7.20(\mathrm{~m}, 3 \mathrm{H}), 5.99$ (ddd, $J=17.3$ and 10.2 and $7.2 \mathrm{~Hz}, 1 \mathrm{H}), 5.10(\mathrm{~m}, 1 \mathrm{H}), 5.06(\mathrm{~m}, 1 \mathrm{H})$, $3.63(\mathrm{~m}, 2 \mathrm{H}), 3.48(\mathrm{q}, J=7.6 \mathrm{~Hz}, 1 \mathrm{H}), 1.98(\mathrm{~m}, 2 \mathrm{H}), 1.41(\mathrm{br} \mathrm{s}, 1 \mathrm{H}) ;{ }^{13} \mathrm{C} \mathrm{NMR}(100$ $\mathrm{MHz}) \delta 143.6,141.7,128.5,127.5,126.3,114.3,60.8,46.2,37.8 ; \mathrm{MS}(\mathrm{m} / \mathrm{z}, \mathrm{EI}) 162$ $\left(\mathrm{M}^{+}\right)$.

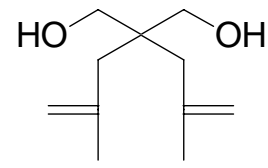

2,2-Bis(2-methylallyl)propane-1,3-diol (Table 2 , entry 6). ${ }^{1}$ The title compound was synthesized in $76 \%$ yield as a colorless oil from reaction of dimethyl malonate and 3bromo-2-methylpropene employing a procedure analogous to the one used to synthesize 2,2-diphenyl-4-hexen-1-ol. ${ }^{1} \mathrm{H}$ NMR (500 MHz) $\delta 4.91(\mathrm{~m}, 2 \mathrm{H}), 4.79(\mathrm{~m}, 2 \mathrm{H}), 3.66(\mathrm{~d}, J$ $=4.7 \mathrm{~Hz}, 4 \mathrm{H}), 2.40(\mathrm{t}, J=4.7 \mathrm{~Hz}, 2 \mathrm{H}), 2.14(\mathrm{~s}, 4 \mathrm{H}), 1.82(\mathrm{~s}, 6 \mathrm{H}) ;{ }^{13} \mathrm{C}$ NMR $(125 \mathrm{MHz}) \delta$ $142.8,115.0,68.0,43.0,40.7,25.2 ; \mathrm{MS}(\mathrm{m} / \mathrm{z}, \mathrm{EI}) 184\left(\mathrm{M}^{+}\right)$.

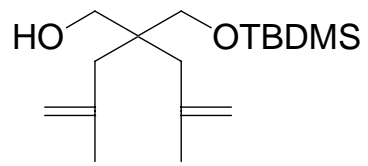


2-(tert-Butyldimethylsiloxymethyl)-4-methyl-2-(methylallyl)-4-penten-1-ol (Table 2, entry 7). ${ }^{1}$ The title compound was prepared in $83 \%$ yield from 2,2-bis(2methylallyl)propane-1,3-diol as a colorless oil according to a known procedure. ${ }^{3}{ }^{1} \mathrm{H}$ NMR (500 MHz) $\delta 4.89(\mathrm{~m}, 2 \mathrm{H}), 4.74(\mathrm{~m}, 2 \mathrm{H}), 3.59(\mathrm{~m}, 4 \mathrm{H}), 2.69(\mathrm{t}, J=6.0 \mathrm{~Hz}, 1 \mathrm{H})$, $2.13(\mathrm{ABq}, J=13.5 \mathrm{~Hz}, 4 \mathrm{H}), 1.79(\mathrm{~s}, 6 \mathrm{H}), 0.90(\mathrm{~s}, 9 \mathrm{H}), 0.06(\mathrm{~s}, 6 \mathrm{H}) ;{ }^{13} \mathrm{C} \mathrm{NMR}(125$ $\mathrm{MHz}) \delta 142.8,115.0,68.8,68.5,43.0,40.6,25.8,25.4,18.1,-5.7$.<smiles>C=C(C)CC(CO)CO</smiles>

2-(2-Methylallyl)propane-1,3-diol (Table 2, entry 8). The title compound was synthesized in $68 \%$ yield as a colorless oil from reaction of dimethyl malonate and 3bromo-2-methylpropene employing a procedure analogous to the one used to synthesize 2,2-diphenyl-4-hexen-1-ol. ${ }^{1} \mathrm{H}$ NMR (400 MHz) $\delta 4.79$ (s, 1H), 4.72 (s, 1H), 3.79-3.75 $(\mathrm{m}, 2 \mathrm{H}), 3.65-3.60(\mathrm{~m}, 2 \mathrm{H}), 3.01(\mathrm{~m}, 3 \mathrm{H}), 1.98(\mathrm{~s}, 3 \mathrm{H}), 1.74(\mathrm{~s}, 3 \mathrm{H}) ;{ }^{13} \mathrm{C}$ NMR $(100$ $\mathrm{MHz}) \delta 143.3,112.2,64.9,39.5,36.5,27.5,22.1 ; \mathrm{MS}(\mathrm{m} / \mathrm{z}, \mathrm{EI}) 130\left(\mathrm{M}^{+}\right)$.<smiles>C=C(C)CC(CO)CO[SnH3]</smiles>

2-(Hydroxymethyl)-4-methylpent-4-enyl triisopropylsilane (Table 2, entry 9). The title compound was prepared in 67\% isolated yield from 2-(2-methylallyl)propane-1,3diol as a colorless oil according to a known procedure. ${ }^{3}{ }^{1} \mathrm{H}$ NMR $(400 \mathrm{MHz}) \delta 4.78(\mathrm{~m}$, $1 \mathrm{H}), 4.71(\mathrm{~m}, 1 \mathrm{H}), 3.90-3.85(\mathrm{~m}, 1 \mathrm{H}), 3.79-3.71(\mathrm{~m}, 1 \mathrm{H}), 3.70-3.63(\mathrm{~m}, 2 \mathrm{H}), 2.58$ (br s, $1 \mathrm{H}), 2.08-1.98(\mathrm{~m}, 1 \mathrm{H}), 1.97-1.95(\mathrm{~m}, 2 \mathrm{H}), 1.74(\mathrm{~s}, 3 \mathrm{H}), 1.10-1.05(\mathrm{~m}, 21 \mathrm{H}) ;{ }^{13} \mathrm{C} \mathrm{NMR}$ $(125 \mathrm{MHz}) \delta 143.4,112.2,67.8,66.9,39.7,36.5,22.3,18.0,11.8 ; \mathrm{MS}(\mathrm{m} / \mathrm{z}, \mathrm{EI}) 286$ $\left(\mathrm{M}^{+}\right)$.<smiles>C=C(C)CC(CO)COC(C)=O</smiles>

2-(Hydroxymethyl)-4-methylpent-4-enyl acetate (Table 2, entry 10). The title compound was prepared in 54\% isolated yield from 2-(2-methylallyl)propane-1,3-diol as pale yellow oil using a procedure adapted from Clarke. ${ }^{4}{ }^{1} \mathrm{H}$ NMR $(500 \mathrm{MHz}) \delta 4.82(\mathrm{~m}$, $1 \mathrm{H}), 4.71(\mathrm{~m}, 1 \mathrm{H}), 4.21-4.18(\mathrm{~m}, 1 \mathrm{H}), 4.09-4.04(\mathrm{~m}, 1 \mathrm{H}), 3.62-3.58(\mathrm{~m}, 1 \mathrm{H}), 3.53-3.47$ $(\mathrm{m}, 1 \mathrm{H}), 2.34$ (br s, 1H), $2.07(\mathrm{~s}, 3 \mathrm{H}), 2.08-2.01(\mathrm{~m}, 3 \mathrm{H}), 1.74(\mathrm{~s}, 3 \mathrm{H}) ;{ }^{13} \mathrm{C}$ NMR $(125$ $\mathrm{MHz}) \delta 171.7,142.8,112.4,64.3,62.2,37.9,36.5,22.0,20.7 ; \mathrm{MS}(\mathrm{m} / \mathrm{z}, \mathrm{EI}) 172\left(\mathrm{M}^{+}\right)$. 
<smiles>C=C(C)CC(CO)COC(=O)OCc1ccccc1</smiles>

2-(Hydroxymethyl)-4-methylpent-4-enyl benzoate (Table 2, entry 11). The title compound was prepared in 50\% isolated yield from 2-(2-methylallyl)propane-1,3-diol using a procedure adapted from Clarke. ${ }^{4} \mathrm{H}$ NMR $(400 \mathrm{MHz}) \delta 7.60-7.57(\mathrm{~m}, 2 \mathrm{H}), 7.56-$ $7.44(\mathrm{~m}, 3 \mathrm{H}), 4.85(\mathrm{~s}, 1 \mathrm{H}), 4.80(\mathrm{~s}, 1 \mathrm{H}), 4.50-4.47(\mathrm{~m}, 1 \mathrm{H}), 4.34-4.3(\mathrm{~m}, 1 \mathrm{H}), 3.69(\mathrm{~m}$, $1 \mathrm{H}), 3.61(\mathrm{~m}, 1 \mathrm{H}), 2.70(\mathrm{br} \mathrm{s}, 1 \mathrm{H}), 2.17(\mathrm{~m}, 3 \mathrm{H}), 1.78(\mathrm{~s}, 3 \mathrm{H}) ;{ }^{13} \mathrm{C}$ NMR $(125 \mathrm{MHz})$ $\delta 167.1,142.8,133.2,129.9,129.6,128.4,112.8,64.5,62.4,38.4,36.7,22.2 ; \mathrm{MS}(\mathrm{m} / \mathrm{z}$, EI) $234\left(\mathrm{M}^{+}\right)$.<smiles>OCC(c1cccc2ccccc12)C1C=CCCC1</smiles>

meso-2-Cyclohexenyl-2-(1-Naphthyl)ethanol (Table 2, entry 12). The title compound was synthesized in $67 \%$ yield as a colorless oil from reaction of 1-naphthaleneacetate and 3-bromocyclohexene employing a procedure analogous to the one used to synthesize 2,2diphenyl-4-hexen-1-ol. ${ }^{1} \mathrm{H}$ NMR $(500 \mathrm{MHz}) \delta 8.16(\mathrm{dd}, J=8.0$ and $7.5 \mathrm{~Hz}, 2 \mathrm{H}), 7.90$ $(\mathrm{dd}, J=7.5$ and $1.5 \mathrm{~Hz}, 2 \mathrm{H}), 7.78(\mathrm{~m}, 2 \mathrm{H}), 7.56-7.47(\mathrm{~m}, 8 \mathrm{H}), 5.95-5.92(\mathrm{~m}, 1 \mathrm{H})$, $5.86-5.82(\mathrm{~m}, 1 \mathrm{H}), 5.65-5.62(\mathrm{~m}, 1 \mathrm{H}), 5.41(\mathrm{~m}, 1 \mathrm{H}), 4.08-4.04(\mathrm{~m}, 4 \mathrm{H}), 3.70(\mathrm{~m}$, 2H), $2.75(\mathrm{~m}, 1 \mathrm{H}), 2.66(\mathrm{~m}, 1 \mathrm{H}), 2.02(\mathrm{~m}, 3 \mathrm{H}), 1.94(\mathrm{~m}, 1 \mathrm{H}), 1.82(\mathrm{~m}, 1 \mathrm{H}), 1.69(\mathrm{~m}, 1 \mathrm{H})$, $1.56(\mathrm{~m}, 2 \mathrm{H}), 1.44(\mathrm{~m}, 3 \mathrm{H}), 1.27(\mathrm{~m}, 1 \mathrm{H}) ;{ }^{13} \mathrm{C} \mathrm{NMR}(125 \mathrm{MHz}) \delta 138.1,137.5,134.1$, 133.0, 130.0, 129.0, 128.9, 128.8, 128.1, 127.1, 127.0, 126.0, 125.5, 125.4, 125.3, 124.1, $123.3,123.2,64.6,64.1,37.9,37.5,27.2,27.1,25.4,25.1,22.0,21.1,20.7 ; \mathrm{MS}(\mathrm{m} / \mathrm{z}, \mathrm{EI})$ $252\left(\mathrm{M}^{+}\right)$.<smiles>CC(C)=CCC(CO)(c1ccccc1)c1ccccc1</smiles>

2,2-Diphenyl-5-methyl-4-hexen-1-ol (Table 2, entry 13). ${ }^{1}$ The title compound was synthesized in $70 \%$ yield as a colorless oil from reaction of methyl diphenylacetate and 3,3-dimethylally bromide employing a procedure analogous to the one used to synthesize 2,2-diphenyl-4-hexen-1-ol. ${ }^{1} \mathrm{H}$ NMR (400 MHz) $\delta 7.33$ - 7.29 (m, 4H), $7.24-7.19$ (m, $6 \mathrm{H}), 4.86(\mathrm{~m}, 1 \mathrm{H}), 4.13(\mathrm{~d}, J=6.8 \mathrm{~Hz}, 2 \mathrm{H}), 2.89(\mathrm{~d}, J=7.2 \mathrm{~Hz}, 2 \mathrm{H}), 1.62(\mathrm{~s}, 3 \mathrm{H}), 1.56$ $(\mathrm{s}, 3 \mathrm{H}), 1.24(\mathrm{t}, J=6.8 \mathrm{~Hz}, 1 \mathrm{H}) ;{ }^{13} \mathrm{C} \mathrm{NMR}(100 \mathrm{MHz}) \delta 145.5,134.5,128.2,128.1$, $126.2,119.7,68.2,53.4,52.2,34.9,25.9,17.8 ; \mathrm{MS}(\mathrm{m} / \mathrm{z}, \mathrm{EI}) 249\left[(\mathrm{M}-\mathrm{OH})^{+}\right]$.

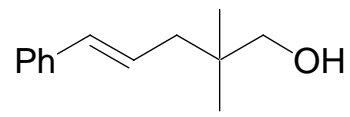


2,2-Dimethyl-5-phenyl-4-penten-1-ol (Table 2, entry 14). The title compound was synthesized in $70 \%$ yield as a colorless oil from reaction of methyl isobutyrate and cinnamyl bromide employing a procedure analogous to the one used to synthesize 2,2diphenyl-4-hexen-1-ol. ${ }^{1} \mathrm{H}$ NMR (500 MHz) $\delta 7.36-7.34$ (m, 2H), $7.30-7.27$ (m, 2H), $7.21-7.18(\mathrm{~m}, 1 \mathrm{H}), 6.40(\mathrm{~d}, J=16.0 \mathrm{~Hz}, 1 \mathrm{H}), 6.28-6.22(\mathrm{~m}, 1 \mathrm{H}), 3.36(\mathrm{~s}, 2 \mathrm{H}), 2.17$ (dd, $J=7.5$ and $1.0 \mathrm{~Hz}, 2 \mathrm{H}), 1.65(\mathrm{br} \mathrm{s}, 1 \mathrm{H}), 0.91(\mathrm{~s}, 6 \mathrm{H}) ;{ }^{13} \mathrm{C}$ NMR $(125 \mathrm{MHz}) \delta 137.6$, $132.3,128.5,127.1,126.9,126.0,71.6,42.3,36.1,23.9$.

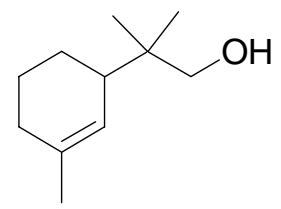

2-(2-Cyclohexenyl-3-methyl)-2,2-dimethylethanol (6). The title compound was synthesized in $70 \%$ yield as a colorless oil from reaction of methyl isobutyrate and 1methyl-3-bromohexene ${ }^{5}$ employing a procedure analogous to the one used to synthesize 2,2-diphenyl-4-hexen-1-ol. ${ }^{1} \mathrm{H}$ NMR $(500 \mathrm{MHz}) \delta 5.40$ (br s, $\left.1 \mathrm{H}\right), 3.47$ (dd, $J=10.9$ and $5.2 \mathrm{~Hz}, 1 \mathrm{H}), 3.38(\mathrm{dd}, J=10.9$ and $6.6 \mathrm{~Hz}, 1 \mathrm{H}), 2.07(\mathrm{~m}, 1 \mathrm{H}), 1.90(\mathrm{~m}, 1 \mathrm{H}), 1.84-1.79$ (m, 2H), $1.74-1.71(\mathrm{~m}, 1 \mathrm{H}), 1.66(\mathrm{~s}, 3 \mathrm{H}), 1.48(\mathrm{~m}, 1 \mathrm{H}), 1.36(\mathrm{t}, J=6.0 \mathrm{~Hz}, 1 \mathrm{H}), 1.17$ $(\mathrm{m}, 1 \mathrm{H}), 0.86(\mathrm{~s}, 6 \mathrm{H}) ;{ }^{13} \mathrm{C}$ NMR $(125 \mathrm{MHz}) \delta 135.4,122.3,70.2,41.4,37.5,29.9,24.2$, 23.6, 23.0, 22.0, 21.6; $\mathrm{MS}(\mathrm{m} / \mathrm{z}, \mathrm{EI}) 168\left(\mathrm{M}^{+}\right)$.<smiles>C=C(C)CC(C(=O)O)c1cccc2ccccc12</smiles>

4-Methyl-2-naphthyl-4-pentenoic acid (Table 4, entry 3). A mixture of 4-methyl-2naphthyl-4-pentenoic acid methyl ester $(1.27 \mathrm{~g}, 5.0 \mathrm{mmol})$ and $\mathrm{NaOH}(1.0 \mathrm{~g}, 25.0 \mathrm{mmol})$ in $20 \mathrm{~mL}$ of methanol and $5 \mathrm{~mL}$ of water was refluxed until reaction was completed. The solvent was removed under vacuum and the residue was dissolved into $20 \mathrm{~mL}$ of water. The resulting mixture was washed with ether $(2 \times 40 \mathrm{~mL})$. The aqueous phase was acidified with $\mathrm{HCl}$ solution to $\mathrm{pH} 1 \sim 2$ and extracted with ether $(3 \times 50 \mathrm{~mL})$. The combined ether phases were washed with brine, dried over $\mathrm{MgSO}_{4}$, and concentrated under vacuum. The residue was subjected to silica gel chromatography to give the title compound (1.08 g, 90\%) as a white solid. ${ }^{1} \mathrm{H}$ NMR $(500 \mathrm{MHz}) \delta 8.21(\mathrm{~d}, J=8.5 \mathrm{~Hz}$, $1 \mathrm{H}), 7.90(\mathrm{~d}, J=7.5 \mathrm{~Hz}, 1 \mathrm{H}), 7.82(\mathrm{~d}, J=8.5 \mathrm{~Hz}, 1 \mathrm{H}), 7.59(\mathrm{~m}, 2 \mathrm{H}), 7.53(\mathrm{~m}, 1 \mathrm{H}), 7.48$ $(\mathrm{t}, J=7.5 \mathrm{~Hz}, 1 \mathrm{H}), 4.83(\mathrm{~s}, 2 \mathrm{H}), 4.72(\mathrm{dd}, J=9.0$ and $6.0 \mathrm{~Hz}, 1 \mathrm{H}), 3.05(\mathrm{dd}, J=15.0$ and $9.0 \mathrm{~Hz}, 1 \mathrm{H}), 2.65(\mathrm{dd}, J=15.0$ and $6.0 \mathrm{~Hz}, 1 \mathrm{H}), 1.80(\mathrm{~s}, 3 \mathrm{H}) ;{ }^{13} \mathrm{C} \mathrm{NMR}(125 \mathrm{MHz}) \delta$ $179.9,142.5,134.3,134.0,131.5,129.0,128.1,126.5,125.7,125.4,125.0,122.9,112.4$, 44.9, 40.5, 22.6; MS (m/z, EI) $240\left(\mathrm{M}^{+}\right)$. 


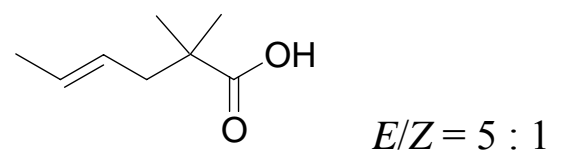

2,2-Dimethyl-4-hexenoic acid (Table 4, entry 4). The title compound was prepared in $69 \%$ yield from reaction of methyl isobutyrate and crotyl bromide followed by hydroxylation employing a procedure analogous to the one used to synthesize 4-methyl2-naphthyl-4-pentenoic acid. For E: ${ }^{1} \mathrm{H}$ NMR $(500 \mathrm{MHz}) \delta 5.50(\mathrm{~m}, 1 \mathrm{H}), 5.40(\mathrm{~m}, 1 \mathrm{H})$, $2.22(\mathrm{~d}, J=7.3 \mathrm{~Hz}, 2 \mathrm{H}), 1.66(\mathrm{dd}, J=5.0$ and $1.0 \mathrm{~Hz}, 3 \mathrm{H}), 1.17(\mathrm{~s}, 6 \mathrm{H}) ;{ }^{13} \mathrm{C} \mathrm{NMR}(125$ MHz) $\delta 184.8,128.8,126.2,43.2,42.4,24.5,18.0$; For $Z:{ }^{1} \mathrm{H}$ NMR (500 MHz) $\delta 5.60$ $(\mathrm{m}, 1 \mathrm{H}), 5.40(\mathrm{~m}, 1 \mathrm{H}), 2.32(\mathrm{~d}, J=7.8 \mathrm{~Hz}, 2 \mathrm{H}), 1.62(\mathrm{dt}, J=5.2$ and $0.8 \mathrm{~Hz}, 3 \mathrm{H}), 1.21(\mathrm{~s}$, $6 \mathrm{H}) ;{ }^{13} \mathrm{C}$ NMR $(125 \mathrm{MHz}) \delta 184.9,126.9,125.4,42.5,37.1,24.5,12.9$.<smiles>CC(C)=CCC(C)(C)C(=O)O</smiles>

2,2,5-Trimethyl-4-hexenoic acid (Table 4, entry 5). ${ }^{6}$ The title compound was prepared in $62 \%$ yield from reaction of methyl isobutyrate and 3,3-dimethylally bromide followed by hydroxylation employing a procedure analogous to the one used to synthesize 4methyl-2-naphthyl-4-pentenoic acid. ${ }^{1} \mathrm{H}$ NMR $(500 \mathrm{MHz}) \delta 5.12(\mathrm{~m}, 1 \mathrm{H}), 2.25(\mathrm{~d}, J=7.6$ $\mathrm{Hz}, 2 \mathrm{H}), 1.71(\mathrm{~s}, 3 \mathrm{H}), 1.61(\mathrm{~s}, 3 \mathrm{H}), 1.18(\mathrm{~s}, 6 \mathrm{H}) ;{ }^{13} \mathrm{C}$ NMR $(125 \mathrm{MHz}) \delta 185.0,134.5$, 119.5, 42.7, 38.4, 26.0, 24.5, 17.9; MS (m/z, EI) $156\left(\mathrm{M}^{+}\right)$.<smiles>C=CC(CC(=O)O)Pc1ccccc1</smiles>

3-Phenyl-4-pentenoic acid (Table 4, entry 6). The title compound was synthesized according to a published procedure. ${ }^{7}{ }^{1} \mathrm{H}$ NMR $(400 \mathrm{MHz}) \delta 7.32-7.28(\mathrm{~m}, 2 \mathrm{H}), 7.22-$ $7.19(\mathrm{~m}, 3 \mathrm{H}), 5.96(\mathrm{~m}, 1 \mathrm{H}), 5.09(\mathrm{~m}, 1 \mathrm{H}), 5.06(\mathrm{dt}, J=5.5$ and $1.3 \mathrm{~Hz}, 1 \mathrm{H}), 3.84(\mathrm{q}, J=$ $7.7 \mathrm{~Hz}, 1 \mathrm{H}), 2.75(\mathrm{~m}, 2 \mathrm{H}) ;{ }^{13} \mathrm{C}$ NMR $(100 \mathrm{MHz}) \delta 178.5,142.1,139.9,128.6,127.5$, 126.8, 115.0, 45.1, 39.9; MS (m/z, EI) $176\left(\mathrm{M}^{+}\right)$.

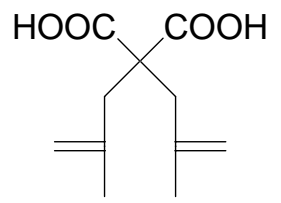

Bis(2-methylallyl)malonic acid (Table 4, entry 7). ${ }^{\mathbf{8}}$ The title compound was synthesized in 56\% yield from reaction of dimethyl malonate and 3-bromo-2methylpropene followed by hydroxylation employing a procedure analogous to the one used to prepare 4-methyl-2-naphthyl-4-pentenoic acid. ${ }^{1} \mathrm{H}$ NMR $(500 \mathrm{MHz}) \delta 4.86(\mathrm{t}, J=$ $1.5 \mathrm{~Hz}, 2 \mathrm{H}), 4.77$ (br s, 2H), $2.76(\mathrm{~s}, 4 \mathrm{H}), 1.71(\mathrm{~s}, 6 \mathrm{H}) ;{ }^{13} \mathrm{C}$ NMR $(125 \mathrm{MHz}) \delta 177.7$, 139.7, 115.5, 56.7, 45.7, 23.1. 
<smiles>O=C(O)C(c1cccc2ccccc12)C1C=CCCC1</smiles>

meso-2-Cyclohexenyl-2-naphthyl acetic acid (Table 4, entry 8). The title compound was synthesized in $66 \%$ yield from reaction of 1-naphthaleneacetate and 3bromocyclohexene followed by hydroxylation employing a procedure analogous to the one used to prepare 4-methyl-2-naphthyl-4-pentenoic acid. ${ }^{1} \mathrm{H}$ NMR (500 MHz) $\delta 8.22$ $(\mathrm{d}, J=8.5 \mathrm{~Hz}, 1 \mathrm{H}), 8.19(\mathrm{~d}, J=8.5 \mathrm{~Hz}, 1 \mathrm{H}), 7.87(\mathrm{~d}, J=8.0 \mathrm{~Hz}, 2 \mathrm{H}), 7.80(\mathrm{~d}, J=8.0 \mathrm{~Hz}$, 2H), $7.72(\mathrm{~d}, J=7.0 \mathrm{~Hz}, 1 \mathrm{H}), 7.66(\mathrm{~d}, \mathrm{~J}=7.0 \mathrm{~Hz}, 1 \mathrm{H}), 7.55(\mathrm{~m}, 2 \mathrm{H}), 7.51-7.46(\mathrm{~m}, 4 \mathrm{H})$, $5.86(\mathrm{~s}, 2 \mathrm{H}), 5.57(\mathrm{~m}, 1 \mathrm{H}), 5.10(\mathrm{~m}, 1 \mathrm{H}), 4.28(\mathrm{~d}, J=11.0 \mathrm{~Hz}, 2 \mathrm{H}), 3.10(\mathrm{~m}, 2 \mathrm{H}), 2.10$ $(\mathrm{m}, 1 \mathrm{H}), 2.00(\mathrm{~m}, 4 \mathrm{H}), 1.86(\mathrm{~m}, 1 \mathrm{H}), 1.66-1.50(\mathrm{~m}, 3 \mathrm{H}), 1.45-1.36(\mathrm{~m}, 2 \mathrm{H}), 1.06-$ $1.00(\mathrm{~m}, 1 \mathrm{H}) ;{ }^{13} \mathrm{C}$ NMR $(125 \mathrm{MHz}) \delta 179.6,179.4,134.02,133.98,133.3,133.0,132.4$, $132.3,129.4,129.2,128.99,128.96,128.90,128.03,127.99,127.6,126.43,126.39$, $125.69,125.61,125.5,125.3,123.3,123.2,51.1,38.3,38.2,28.2,26.0,25.3,25.2,21.2$, 20.9 .<smiles>C=CCCC(C)(C)C(=O)O</smiles>

2,2-Dimethyl-5-hexenoic acid (Table 4, entry 9). ${ }^{\mathbf{9}}$ The title compound was synthesized in $64 \%$ yield from reaction of methyl isobutyrate and 4-bromobutene followed by hydroxylation employing a procedure analogous to the one used to prepare 4-methyl-2naphthyl-4-pentenoic acid. ${ }^{1} \mathrm{H}$ NMR $(500 \mathrm{MHz}) \delta 5.79(\mathrm{~m}, 1 \mathrm{H}), 5.02(\mathrm{~m}, 1 \mathrm{H}), 4.94(\mathrm{~m}$, $1 \mathrm{H}), 2.05(\mathrm{~m}, 2 \mathrm{H}), 1.64(\mathrm{~m}, 2 \mathrm{H}), 1.21(\mathrm{~s}, 6 \mathrm{H}) ;{ }^{13} \mathrm{C} \mathrm{NMR}(125 \mathrm{MHz}) \delta 185.0,138.2$, 114.6, 41.9, 39.5, 29.2, 24.9; $\mathrm{MS}(\mathrm{m} / \mathrm{z}, \mathrm{EI}) 142\left(\mathrm{M}^{+}\right)$.<smiles>CC1=CC(C(C)(C)C(=O)O)CCC1</smiles>

$\boldsymbol{\alpha , \alpha , 3 - T r i m e t h y l - 2 - c y c l o h e x e n e - 1 - a c e t i c ~ a c i d ~ ( 9 ) . ~ T h e ~ t i t l e ~ c o m p o u n d ~ w a s ~ s y n t h e s i z e d ~}$ in $70 \%$ yield as colorless oil from reaction of methyl isobutyrate and 1-methyl-3bromohexene ${ }^{5}$ followed by hydroxylation employing a procedure analogous to the one used to prepare 4-methyl-2-naphthyl-4-pentenoic acid. ${ }^{1} \mathrm{H}$ NMR $(500 \mathrm{MHz}) \delta 5.21$ (br s, $1 \mathrm{H}), 2.48(\mathrm{~m}, 1 \mathrm{H}), 1.89(\mathrm{~m}, 1 \mathrm{H}), 1.85-1.79(\mathrm{~m}, 2 \mathrm{H}), 1.68(\mathrm{~m}, 1 \mathrm{H}), 1.65(\mathrm{~s}, 3 \mathrm{H}), 1.52-$ $1.48(\mathrm{~m}, 1 \mathrm{H}), 1.18(\mathrm{~m}, 1 \mathrm{H}), 1.14(\mathrm{~s}, 3 \mathrm{H}), 1.10(\mathrm{~s}, 3 \mathrm{H}) ;{ }^{13} \mathrm{C} \mathrm{NMR}(125 \mathrm{MHz}) \delta 185.2$, $136.4,121.5,45.3,42.9,30.0,24.0,23.9,22.6,21.5$.

General procedure for the silver triflate catalyzed intramolecular cyclization: A mixture of hydroxyl-olefin or carboxyl-olefin $(1.0 \mathrm{mmol})$ and silver triflate $(13.0 \mathrm{mg}$, 
$0.05 \mathrm{mmol}$ ) in $2 \mathrm{~mL}$ of dry 1,2-dichloroethane was heating at $83{ }^{\circ} \mathrm{C}$ for $15-36 \mathrm{~h}$ in a dry sealed tube under nitrogen atmosphere. The reaction was cooled to $\mathrm{rt}$ and the catalyst was removed by filtration through a short pad of silica gel. The ether eluant was then concentrated and the residue was subjected to silica gel chromatography to give the corresponding cyclic ethers or lactones.<smiles>CC1CCC(c2ccccc2)(c2ccccc2)CO1</smiles>

2-Methyl-5,5-diphenyltetrahydropyran (Table 2, entry 3). ${ }^{1}{ }^{1} \mathrm{H}$ NMR (500 MHz) $\delta$ $7.43-7.41(\mathrm{~m}, 2 \mathrm{H}), 7.28-7.23(\mathrm{~m}, 4 \mathrm{H}), 7.17-7.16(\mathrm{~m}, 4 \mathrm{H}), 4.63(\mathrm{dd}, J=12.5$ and 3.0 $\mathrm{Hz}, 1 \mathrm{H}), 3.56(\mathrm{~d}, J=12.5 \mathrm{~Hz}, 1 \mathrm{H}), 3.56(\mathrm{~m}, 1 \mathrm{H}), 2.48-2.38(\mathrm{~m}, 2 \mathrm{H}), 1.54(\mathrm{dq}, J=11.0$ and $3.5 \mathrm{~Hz}, 1 \mathrm{H}), 1.29-1.18(\mathrm{~m}, 1 \mathrm{H}), 1.16(\mathrm{~d}, J=6.0 \mathrm{~Hz}, 3 \mathrm{H}) ;{ }^{13} \mathrm{C} \mathrm{NMR}(125 \mathrm{MHz}) \delta$ 146.7, 145.8, 128.9, 128.2, 127.9, 126.9, 126.2, 125.5, 75.0, 73.9, 45.7, 34.7, 29.7, 21.6; $\mathrm{MS}(\mathrm{m} / \mathrm{z}, \mathrm{EI}) 252\left(\mathrm{M}^{+}\right)$.

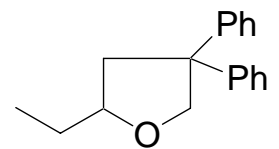

2-Ethyl-4,4-diphenyltetrahydrofuran (Table 2, Entry 3). ${ }^{1}{ }^{1} \mathrm{H}$ NMR (400 MHz) $\delta 7.33$ $-7.18(\mathrm{~m}, 10 \mathrm{H}), 4.62(\mathrm{dd}, J=8.7$ and $0.8 \mathrm{~Hz}, 1 \mathrm{H}), 4.12(\mathrm{~d}, J=8.7 \mathrm{~Hz}, 1 \mathrm{H}), 3.96(\mathrm{~m}$, 1H), 2.60 (ddd, $J=12.0$ and 5.7 and $1.0 \mathrm{~Hz}, 1 \mathrm{H}$ ), 2.28 (dd, $J=12.0$ and $9.6 \mathrm{~Hz}, 1 \mathrm{H}$ ), $1.68(\mathrm{~m}, 1 \mathrm{H}), 1.56(\mathrm{~m}, 1 \mathrm{H}), 0.94(\mathrm{t}, J=7.5 \mathrm{~Hz}, 3 \mathrm{H}) ;{ }^{13} \mathrm{C} \mathrm{NMR}(100 \mathrm{MHz}) \delta 146.4$, $146.0,128.3,128.2,127.1,126.3,126.1,80.1,76.7,55.9,44.4,28.9,10.3 ; \mathrm{MS}(\mathrm{m} / \mathrm{z}, \mathrm{EI})$ $252\left(\mathrm{M}^{+}\right)$.

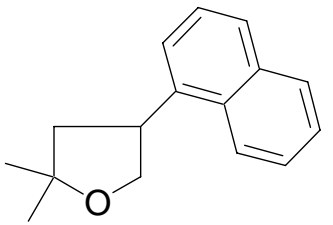

2,2-Dimethyl-4-naphthyltetrahydrofuran (Table 2, entry 4). ${ }^{1} \mathrm{H}$ NMR (400 MHz) $\delta$ $8.16(\mathrm{~d}, J=8.5 \mathrm{~Hz}, 1 \mathrm{H}), 7.89(\mathrm{dd}, J=7.9$ and $1.5 \mathrm{~Hz}, 1 \mathrm{H}), 7.76(\mathrm{~d}, J=7.9 \mathrm{~Hz}, 1 \mathrm{H}), 7.58$ $-7.44(\mathrm{~m}, 4 \mathrm{H}), 4.42(\mathrm{dd}, J=8.3$ and $7.5 \mathrm{~Hz}, 1 \mathrm{H}), 4.30(\mathrm{~m}, 1 \mathrm{H}), 4.02(\mathrm{dd}, J=8.3$ and 7.5 $\mathrm{Hz}, 1 \mathrm{H}), 2.36(\mathrm{dd}, J=12.3$ and $7.9 \mathrm{~Hz}, 1 \mathrm{H}), 2.10(\mathrm{dd}, J=12.3$ and $9.7 \mathrm{~Hz}, 1 \mathrm{H}), 1.44(\mathrm{~s}$, $6 \mathrm{H}) ;{ }^{13} \mathrm{C}$ NMR $(100 \mathrm{MHz}) \delta 137.8,133.9,132.0,128.9,127.0,125.9,125.5,123.4$, 122.7, 81.1, 72.4, 45.8, 41.2, 28.9, 28.4; MS (m/z, EI) $226\left(\mathrm{M}^{+}\right)$; Cald for $\mathrm{C}_{16} \mathrm{H}_{18} \mathrm{O}$ : C, 84.91, H, 8.02; found C, 84.88, H, 7.94.

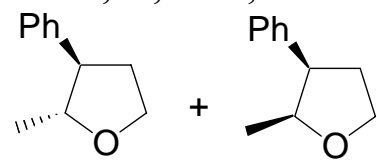

2-Methyl-3-phenyl-tetrahydrofuran (Table 2, entry 5). Trans : cis $=2.7: \mathbf{1}^{1}{ }^{1} \mathrm{H}$ NMR $(500 \mathrm{MHz}) \delta 7.33-7.15(\mathrm{~m}, 5 \mathrm{H}),[4.19-4.13(\mathrm{~m}), 4.06-4.00(\mathrm{~m}), 2 \mathrm{H}, 1: 3], 3.89-$ $3.83(\mathrm{~m}, 1 \mathrm{H}),[3.32(\mathrm{~m}), 2.80(\mathrm{q}, \mathrm{J}=8.9 \mathrm{~Hz}), 1 \mathrm{H}, 1: 3], 2.42-2.35(\mathrm{~m}, 1 \mathrm{H}), 2.21-2.06$ $(\mathrm{m}, 1 \mathrm{H}),[1.22(\mathrm{~d}, \mathrm{~J}=6.0 \mathrm{~Hz}), 0.84(\mathrm{~d}, \mathrm{~J}=6.4 \mathrm{~Hz}), 3 \mathrm{H}, 3: 1]$; For trans: ${ }^{13} \mathrm{C}$ NMR $(125$ 
MHz) $\delta 141.4,128.5,127.5,126.5,82.1,67.3,52.9,35.3,18.9$; For cis: 141.7, 128.3, $128.1,126.2,78.1,66.8,48.3,32.7,16.8 ; \mathrm{MS}(\mathrm{m} / \mathrm{z}, \mathrm{EI}) 162\left(\mathrm{M}^{+}\right)$.

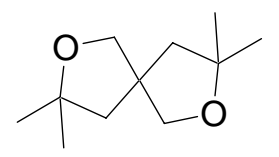

3,3,8,8-Tetramethyl-2,7-dioxaspiro[4.4]nonane (Table 2, entry 6). ${ }^{1}{ }^{1} \mathrm{H}$ NMR (500 $\mathrm{MHz}) \delta 3.80(\mathrm{~d}, J=8.5 \mathrm{~Hz}, 2 \mathrm{H}), 3.61(\mathrm{~d}, J=8.5 \mathrm{~Hz}, 2 \mathrm{H}), 1.79(\mathrm{~s}, 4 \mathrm{H}), 1.22(\mathrm{~s}, 6 \mathrm{H}), 1.19$ $(\mathrm{s}, 6 \mathrm{H}) ;{ }^{13} \mathrm{C}$ NMR $(125 \mathrm{MHz}) \delta 80.2,76.5,52.8,49.3,28.9,28.4 ; \mathrm{MS}(\mathrm{m} / \mathrm{z}, \mathrm{EI}) 184\left(\mathrm{M}^{+}\right)$.

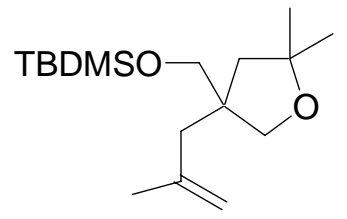

(1,1-Dimethylethyl)dimethyl[[tetrahydro-5,5-dimethyl-3-(2-methyl-2-propenyl)-3-

furanyl]methyl]silane (Table 2, entry 7). ${ }^{1} \mathrm{H}$ NMR $(500 \mathrm{MHz}) \delta 4.82(\mathrm{~m}, 1 \mathrm{H}), 4.70$ $(\mathrm{m}, 1 \mathrm{H}), 3.72$ (d, $J=9.0 \mathrm{~Hz}, 1 \mathrm{H}), 3.51(\mathrm{~d}, J=9.0 \mathrm{~Hz}, 1 \mathrm{H}), 3.46(\mathrm{~d}, J=9.5 \mathrm{~Hz}, 1 \mathrm{H}), 3.40$ $(\mathrm{d}, J=9.5 \mathrm{~Hz}, 1 \mathrm{H}), 2.24,2.16(\mathrm{ABq}, J=13.4 \mathrm{~Hz}, 2 \mathrm{H}), 1.73(\mathrm{br} \mathrm{s}, 3 \mathrm{H}), 1.63,1.59(\mathrm{ABq}$, $J=13.4 \mathrm{~Hz}, 2 \mathrm{H}), 1.25(\mathrm{~s}, 3 \mathrm{H}), 1.24(\mathrm{~s}, 3 \mathrm{H}), 0.89(\mathrm{~s}, 6 \mathrm{H}), 0.030(\mathrm{~s}, 3 \mathrm{H}), 0.024(\mathrm{~s}, 3 \mathrm{H}) ;{ }^{13} \mathrm{C}$ NMR $(125 \mathrm{MHz}) \delta 143.0,114.1,80.5,73.5,65.5,49.8,46.5,42.6,29.4,28.7,25.8,24.4$, $18.2,-5.6$.<smiles>CC1(C)CC(CO)CO1</smiles>

(Tetrahydro-5,5-dimethylfuran-3-yl)methanol (Table 2, entry 8). ${ }^{1} \mathrm{H}$ NMR (500 MHz) $\delta 3.97(\mathrm{dd}, J=9.0$ and $7.5 \mathrm{~Hz}, 1 \mathrm{H}), 3.67-3.57(\mathrm{~m}, 3 \mathrm{H}), 2.57(\mathrm{~m}, 1 \mathrm{H}), 2.05$ (br s, $1 \mathrm{H}), 1.90(\mathrm{dd}, J=12.5$ and $7.5 \mathrm{~Hz}, 1 \mathrm{H}), 1.42(\mathrm{dd}, J=12.5$ and $7.5 \mathrm{~Hz}, 1 \mathrm{H}), 1.30(\mathrm{~s}, 3 \mathrm{H})$, $1.21(\mathrm{~s}, 3 \mathrm{H}) ;{ }^{13} \mathrm{C} \mathrm{NMR}(125 \mathrm{MHz}) \delta 81.1,69.5,65.0,42.4,41.5,28.7,27.6 ; \mathrm{MS}(\mathrm{m} / \mathrm{z}, \mathrm{EI})$ $130\left(\mathrm{M}^{+}\right)$.

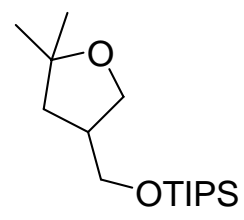

(Tetrahydro-5,5-dimethylfuran-3-yl)methoxytriisopropylsilane (Table 2, entry 9). ${ }^{1} \mathrm{H}$ NMR $(400 \mathrm{MHz}) \delta 3.94(\mathrm{dd}, J=8.8$ and $7.6 \mathrm{~Hz}, 1 \mathrm{H}), 3.70-3.60(\mathrm{~m}, 3 \mathrm{H}), 2.57(\mathrm{~m}, 1 \mathrm{H})$, $1.83(\mathrm{dd}, J=12.4$ and $8.4 \mathrm{~Hz}, 1 \mathrm{H}), 1.45(\mathrm{dd}, J=12.4$ and $8.4 \mathrm{~Hz}, 1 \mathrm{H}), 1.29(\mathrm{~s}, 3 \mathrm{H}), 1.21$ 
(s, 3H), 1.12-1.03 (m, 21H); ${ }^{13} \mathrm{C}$ NMR $(125 \mathrm{MHz}) \delta 80.8,69.6,65.5,42.9,41.4,28.8$, 27.9, 18.1, 12.1; MS (m/z, EI) $286\left(\mathrm{M}^{+}\right)$.

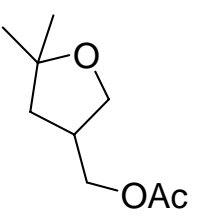

(Tetrahydro-5,5-dimethylfuran-3-yl)methyl acetate (Table 2, entry 10). ${ }^{1} \mathrm{H}$ NMR $(500 \mathrm{MHz}) \delta 4.11(\mathrm{dd}, J=11.0$ and $6.0 \mathrm{~Hz}, 1 \mathrm{H}), 4.00-3.95(\mathrm{~m}, 2 \mathrm{H}), 3.61(\mathrm{dd}, J=9.0$ and $7.0 \mathrm{~Hz}, 1 \mathrm{H}), 2.68(\mathrm{~m}, 1 \mathrm{H}), 2.06(\mathrm{~s}, 3 \mathrm{H}), 1.93(\mathrm{dd}, J=12.5$ and $8.0 \mathrm{~Hz}, 1 \mathrm{H}), 1.44(\mathrm{dd}, J=$ 12.5 and $8.0 \mathrm{~Hz}, 1 \mathrm{H}), 1.30(\mathrm{~s}, 3 \mathrm{H}), 1.22(\mathrm{~s}, 3 \mathrm{H}) ;{ }^{13} \mathrm{C} \mathrm{NMR}(125 \mathrm{MHz}) \delta 170.8,80.6$, 69.1, 66.0, 41.4, 38.9, 28.4, 27.5, 20.7; MS (m/z, EI) $172\left(\mathrm{M}^{+}\right)$.

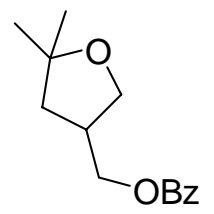

(Tetrahydro-5,5-dimethylfuran-3-yl)methyl benzoate (Table 2, entry 11). ${ }^{1} \mathrm{H}$ NMR $(500 \mathrm{MHz}) \delta 8.03-8.00(\mathrm{~m}, 2 \mathrm{H}), 7.57-7.54(\mathrm{~m}, 1 \mathrm{H}), 7.45-7.41(\mathrm{~m}, 1 \mathrm{H}), 4.34(\mathrm{dd}, J=11.0$ and $6.5 \mathrm{~Hz}, 1 \mathrm{H}), 4.23(\mathrm{dd}, J=10.5$ and $8.0 \mathrm{~Hz}, 1 \mathrm{H}), 4.03(\mathrm{dd}, J=8.5$ and $7.0 \mathrm{~Hz}, 1 \mathrm{H})$, $3.71(\mathrm{dd}, J=8.5$ and $7.0 \mathrm{~Hz}, 1 \mathrm{H}), 2.83(\mathrm{~m}, 1 \mathrm{H}), 1.98(\mathrm{dd}, J=12.5$ and $8.0 \mathrm{~Hz}, 1 \mathrm{H}), 1.53$ (dd, $J=12.5$ and $8.0 \mathrm{~Hz}, 1 \mathrm{H}), 1.32(\mathrm{~s}, 3 \mathrm{H}), 1.23(\mathrm{~s}, 3 \mathrm{H}) ;{ }^{13} \mathrm{C}$ NMR $(125 \mathrm{MHz}) \delta 166.3$, 132.9, 130.0, 129.4, 128.3, 80.8, 69.2, 66.5, 41.6, 39.2, 28.6, 27.5; MS (m/z, EI) 234 $\left(\mathrm{M}^{+}\right)$.

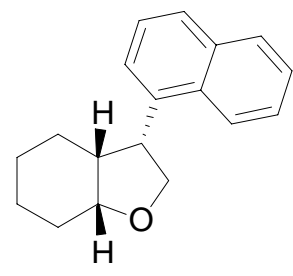

endo-cis-3-Naphthyloctahydrobenzofuran (Table 2, entry 12). ${ }^{1} \mathrm{H}$ NMR $(500 \mathrm{MHz}) \delta$ $8.08(\mathrm{~d}, J=8.5 \mathrm{~Hz}, 1 \mathrm{H}), 7.88(\mathrm{~d}, J=8.0 \mathrm{~Hz}, 1 \mathrm{H}), 7.75(\mathrm{~d}, J=7.5 \mathrm{~Hz}, 1 \mathrm{H}), 7.57-7.45$ $(\mathrm{m}, 4 \mathrm{H}), 4.55(\mathrm{dd}, J=9.0$ and $8.0 \mathrm{~Hz}, 1 \mathrm{H}), 4.20(\mathrm{q}, J=4.5 \mathrm{~Hz}, 1 \mathrm{H}), 4.17$ (dd, $J=9.0$ and $4.0 \mathrm{~Hz}, 1 \mathrm{H}), 3.99(\mathrm{dt}, J=8.0$ and $4.0 \mathrm{~Hz}, 1 \mathrm{H}), 2.29-2.25(\mathrm{~m}, 1 \mathrm{H}), 2.00-1.91(\mathrm{~m}, 2 \mathrm{H})$, $1.70-1.66(\mathrm{~m}, 1 \mathrm{H}), 1.63-1.54(\mathrm{~m}, 3 \mathrm{H}), 1.46-1.43(\mathrm{~m}, 1 \mathrm{H}), 1.29-1.26(\mathrm{~m}, 1 \mathrm{H}) ;{ }^{13} \mathrm{C}$ NMR (125 MHz) $\delta 138.8,134.0,131.9,129.0,126.9,126.0,125.5,125.4,123.2,123.0$, 76.3, 71.9, 45.6, 44.7, 28.5, 27.6, 23.7, 21.1; MS (m/z, EI) $252\left(\mathrm{M}^{+}\right)$.

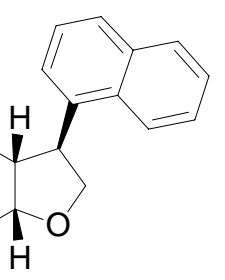


exo-cis-3-Naphthyloctahydrobenzofuran (Table 2, entry 12). ${ }^{1} \mathrm{H}$ NMR $(500 \mathrm{MHz}) \delta$ $8.15(\mathrm{~d}, J=8.0 \mathrm{~Hz}, 1 \mathrm{H}), 7.87(\mathrm{~d}, J=8.5 \mathrm{~Hz}, 1 \mathrm{H}), 7.75(\mathrm{~d}, J=8.5 \mathrm{~Hz}, 1 \mathrm{H}), 7.56-7.49$ $(\mathrm{m}, 2 \mathrm{H}), 7.42(\mathrm{~m}, 1 \mathrm{H}), 7.37(\mathrm{~d}, J=6.0 \mathrm{~Hz}, 1 \mathrm{H}), 4.60(\mathrm{dd}, J=10.0$ and $8.5 \mathrm{~Hz}, 1 \mathrm{H}), 4.42$ (td, $J=8.5$ and $3.5 \mathrm{~Hz}, 1 \mathrm{H}), 4.36(\mathrm{q}, J=3.0 \mathrm{~Hz}, 1 \mathrm{H}), 4.24(\mathrm{t}, J=8.5 \mathrm{~Hz}, 1 \mathrm{H}), 2.49-2.44$ $(\mathrm{m}, 1 \mathrm{H}), 2.11-2.08(\mathrm{~m}, 1 \mathrm{H}), 1.62-1.54(\mathrm{~m}, 2 \mathrm{H}), 1.47-1.42(\mathrm{~m}, 2 \mathrm{H}), 1.08-0.99(\mathrm{~m}$, $2 \mathrm{H}), 0.66-0.63(\mathrm{~m}, 1 \mathrm{H}) ;{ }^{13} \mathrm{C}$ NMR $(125 \mathrm{MHz}) \delta 133.8,133.4,132.5,128.9,127.1$, $125.9,125.5,125.0,123.6,123.2,78.7,68.2,45.1,41.6,28.8,24.2,23.1,20.4 ; \mathrm{MS}(\mathrm{m} / \mathrm{z}$, EI) $252\left(\mathrm{M}^{+}\right)$.<smiles>CC1(C)CCC(c2ccccc2)(c2ccccc2)CO1</smiles>

2,2-Dimethyl-5,5-diphenyltetrahydropyran (Table 2, entry 13). ${ }^{1} \mathrm{H}$ NMR $(500 \mathrm{MHz})$ $\delta 7.35-7.34(\mathrm{~m}, 4 \mathrm{H}), 7.31-7.28(\mathrm{~m}, 4 \mathrm{H}), 7.21-7.18(\mathrm{~m}, 2 \mathrm{H}), 4.09(\mathrm{~s}, 2 \mathrm{H}), 2.46(\mathrm{t}, J=$ $6.0 \mathrm{~Hz}, 2 \mathrm{H}), 1.43(\mathrm{t}, J=6.0 \mathrm{~Hz}, 2 \mathrm{H}), 1.26(\mathrm{~s}, 6 \mathrm{H}) ;{ }^{13} \mathrm{C} \mathrm{NMR}(125 \mathrm{MHz}) \delta 146.4,128.0$, $127.8,125.9,71.2,68.9,45.8,32.5,30.7,26.3 ; \mathrm{MS}(\mathrm{m} / \mathrm{z}, \mathrm{EI}) 266\left(\mathrm{M}^{+}\right)$.<smiles>CC1(C)CCC(c2ccccc2)OC1</smiles>

2-Phenyl-5,5-dimethyltetrahydropyran (Table 2, entry 14). ${ }^{1} \mathrm{H}$ NMR $(500 \mathrm{MHz})$ $\delta 7.38-7.31(\mathrm{~m}, 4 \mathrm{H}), 7.27-7.23(\mathrm{~m}, 1 \mathrm{H}), 4.23(\mathrm{dd}, J=11.0$ and $0.8 \mathrm{~Hz}, 1 \mathrm{H}), 3.62(\mathrm{dd}$, $J=11.0$ and $0.8 \mathrm{~Hz}, 1 \mathrm{H}), 3.34(\mathrm{~d}, J=11.0 \mathrm{~Hz}, 1 \mathrm{H}), 1.84-1.75(\mathrm{~m}, 1 \mathrm{H}), 1.70-1.66(\mathrm{~m}$, $1 \mathrm{H}), 1.63-1.58(\mathrm{~m}, 1 \mathrm{H}), 1.56-1.49(\mathrm{~m}, 1 \mathrm{H}) 1.13(\mathrm{~s}, 3 \mathrm{H}), 0.87(\mathrm{~s}, 3 \mathrm{H}) ;{ }^{13} \mathrm{C} \mathrm{NMR}(125$ $\mathrm{MHz}) \delta 143.1,128.2,127.2,125.8,80.2,78.6,37.2,30.4,29.8,27.2,23.4$.

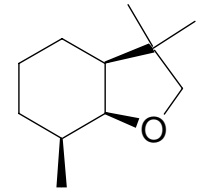

cis,cis-3,3,7-Trimethyloctahydrobenzofuran (7). ${ }^{1} \mathrm{H}$ NMR $(500 \mathrm{MHz}) \delta 4.06$ (dd, $J=$ 3.5 and $3.0 \mathrm{~Hz}, 1 \mathrm{H}), 3.63(\mathrm{~d}, J=8.0 \mathrm{~Hz}, 1 \mathrm{H}), 3.51(\mathrm{~d}, J=8.0 \mathrm{~Hz}, 1 \mathrm{H}), 1.74-1.71(\mathrm{~m}$, $1 \mathrm{H}), 1.61-1.53(\mathrm{~m}, 2 \mathrm{H}), 1.48-1.44(\mathrm{~m}, 1 \mathrm{H}), 1.41-1.38(\mathrm{~m}, 1 \mathrm{H}), 1.19-1.11(\mathrm{~m}, 2 \mathrm{H})$, $1.13(\mathrm{~s}, 3 \mathrm{H}), 1.09-1.02(\mathrm{~m}, 1 \mathrm{H}), 1.03(\mathrm{~d}, J=7.0 \mathrm{~Hz}, 3 \mathrm{H}), 0.98(\mathrm{~s}, 3 \mathrm{H}) ;{ }^{13} \mathrm{C} \mathrm{NMR}(125$ $\mathrm{MHz}) \delta 80.7,78.3,48.2,41.6,34.6,28.5,25.1,24.4,21.4,18.9 ; \mathrm{MS}(\mathrm{m} / \mathrm{z}, \mathrm{EI}) 168\left(\mathrm{M}^{+}\right)$.

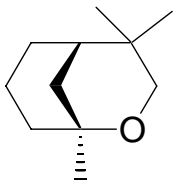


1,4,4-Trimethyl-2-oxabicyclo[3.3.1]nonan (8). ${ }^{1} \mathrm{H}$ NMR $(500 \mathrm{MHz}) \delta 3.90(\mathrm{~d}, J=12.0$ $\mathrm{Hz}, 1 \mathrm{H}), 3.31(\mathrm{~d}, J=12.0 \mathrm{~Hz}, 1 \mathrm{H}), 2.0-1.9(\mathrm{~m}, 3 \mathrm{H}), 1.87-1.83(\mathrm{~m}, 1 \mathrm{H}), 1.61-1.56$ $(\mathrm{m}, 2 \mathrm{H}), 1.50-1.42(\mathrm{~m}, 2 \mathrm{H}), 1.27(\mathrm{~m}, 1 \mathrm{H}), 1.10(\mathrm{~s}, 3 \mathrm{H}), 1.08(\mathrm{~s}, 3 \mathrm{H}), 0.90(\mathrm{~s}, 3 \mathrm{H}) ;{ }^{13} \mathrm{C}$ NMR $(125 \mathrm{MHz}) \delta 74.0,69.8,37.7,36.9,36.4,32.8,30.2,28.6,26.9,24.4,21.1$; MS $(\mathrm{m} / \mathrm{z}, \mathrm{EI}) 168\left(\mathrm{M}^{+}\right)$.

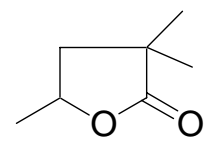

Dihydro-3,3,5-trimethyl-2(3H)-furanone (Table 4, entry 2). ${ }^{\mathbf{1 0}} \mathrm{mp} 49-50{ }^{\circ} \mathrm{C} ;{ }^{1} \mathrm{H}$ NMR $(500 \mathrm{MHz}) \delta 4.56(\mathrm{~m}, 1 \mathrm{H}), 2.18(\mathrm{dd}, J=12.7$ and $5.8 \mathrm{~Hz}, 1 \mathrm{H}), 1.70(\mathrm{dd}, J=12.7$ and 9.9 $\mathrm{Hz}, 1 \mathrm{H}), 1.40(\mathrm{~d}, J=6.1 \mathrm{~Hz}, 3 \mathrm{H}), 1.27(\mathrm{~s}, 3 \mathrm{H}), 1.25(\mathrm{~s}, 3 \mathrm{H}) ;{ }^{13} \mathrm{C}$ NMR $(125 \mathrm{MHz}) \delta$ 181.9, 73.3, 45.1, 40.8, 25.0, 24.2, 21.0; $\mathrm{MS}(\mathrm{m} / \mathrm{z}, \mathrm{EI}) 128\left(\mathrm{M}^{+}\right)$.

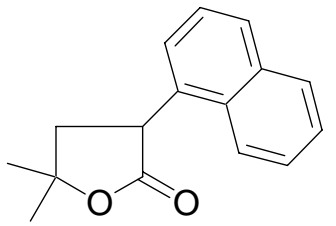

Dihydro-5,5-dimethyl-3-(1-naphthyl)-2(3H)-furanone (Table 4, entry 3). mp 112 $114{ }^{\circ} \mathrm{C} ;{ }^{1} \mathrm{H}$ NMR $(400 \mathrm{MHz}) \delta 7.91-7.86(\mathrm{~m}, 2 \mathrm{H}), 7.83-7.80(\mathrm{~m}, 1 \mathrm{H}), 7.57-7.49(\mathrm{~m}$, $2 \mathrm{H}), 7.47-7.46(\mathrm{~m}, 2 \mathrm{H}), 4.72(\mathrm{dd}, J=10.5$ and $9.6 \mathrm{~Hz}, 1 \mathrm{H}), 2.73(\mathrm{dd}, J=12.9$ and 9.6 $\mathrm{Hz}, 1 \mathrm{H}), 2.29(\mathrm{dd}, J=12.9$ and $10.5 \mathrm{~Hz}, 1 \mathrm{H}), 1.58(\mathrm{~s}, 3 \mathrm{H}), 1.56(\mathrm{~s}, 3 \mathrm{H}) ;{ }^{13} \mathrm{C}$ NMR $(100$ $\mathrm{MHz}) \delta 176.7,134.0,133.6,131.2,129.2,128.3,126.4,125.8,125.6,122.8,82.5,44.5$, 44.0, 28.9, 27.5; MS (m/z, EI) $240\left(\mathrm{M}^{+}\right)$.

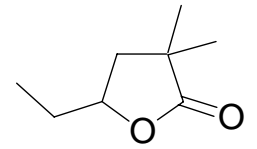

Dihydro-5-ethyl-3,3-dimethyl-2(3H)-furanone (Table 4, entries 4 and 9). ${ }^{10}{ }^{1} \mathrm{H}$ NMR $(500 \mathrm{MHz}) \delta 4.31(\mathrm{~m}, 1 \mathrm{H}), 2.11(\mathrm{dd}, J=12.7$ and $6.0 \mathrm{~Hz}, 1 \mathrm{H}), 1.70(\mathrm{~m}, 1 \mathrm{H}), 1.68(\mathrm{dd}, J$ $=12.7$ and $10.0 \mathrm{~Hz}, 1 \mathrm{H}), 1.58(\mathrm{~m}, 1 \mathrm{H}), 1.22(\mathrm{~s}, 3 \mathrm{H}), 1.21(\mathrm{~s}, 3 \mathrm{H}), 0.95(\mathrm{t}, J=7.4 \mathrm{~Hz}$, $3 \mathrm{H}) ;{ }^{13} \mathrm{C}$ NMR $(125 \mathrm{MHz}) \delta 181.9,78.2,42.9,40.3,28.5,24.9,24.3,9.4 ; \mathrm{MS}(\mathrm{m} / \mathrm{z}, \mathrm{EI})$ $142\left(\mathrm{M}^{+}\right)$.<smiles>CC1CCC(C)(C)C(=O)O1</smiles>

Tetrahydro-3,3,6-trimethyl-2H-pyran-2-one (Table 4, entries 4 and 9). ${ }^{11}{ }^{1} \mathrm{H}$ NMR $(500 \mathrm{MHz}) \delta 4.37(\mathrm{~m}, 1 \mathrm{H}), 1.79-1.75(\mathrm{~m}, 1 \mathrm{H}), 1.72-1.56(\mathrm{~m}, 3 \mathrm{H}), 1.28(\mathrm{~d}, J=6.5 \mathrm{~Hz}$, $3 \mathrm{H}), 1.20(\mathrm{~s}, 3 \mathrm{H}), 1.19(\mathrm{~s}, 3 \mathrm{H}) ;{ }^{13} \mathrm{C}$ NMR $(125 \mathrm{MHz}) \delta 177.4,77.8,37.5,34.2,27.6,27.5$, 21.8; MS (m/z, EI) $142\left(\mathrm{M}^{+}\right)$.<smiles>CC1(C)CCC(C)(C)C(=O)O1</smiles> 
Tetrahydro-3,3,6,6-tetramethyl-2H-pyran-2-one (Table 4, entry 5). ${ }^{12} \mathrm{mp} 79-80{ }^{\circ} \mathrm{C}$; ${ }^{1} \mathrm{H}$ NMR $(500 \mathrm{MHz}) \delta 1.82-1.79(\mathrm{~m}, 2 \mathrm{H}), 1.76-1.74(\mathrm{~m}, 2 \mathrm{H}), 1.38(\mathrm{~s}, 6 \mathrm{H}), 1.27(\mathrm{~s}$, $6 \mathrm{H}) ;{ }^{13} \mathrm{C}$ NMR $(125 \mathrm{MHz}) \delta 177.6,82.4,37.1,32.0,31.5,28.9,27.6$; MS (m/z, EI) 156 $\left(\mathrm{M}^{+}\right)$.<smiles>C[C@@H]1OC(=O)C[C@@H]1Pc1ccccc1</smiles>

trans-Dihydro-5-methyl-4-phenyl-2(3H)-furanone (Table 4, entry 6). ${ }^{13} \mathrm{H}$ NMR (400 MHz) $\delta 7.38(\mathrm{~m}, 2 \mathrm{H}), 7.30(\mathrm{~m}, 1 \mathrm{H}), 7.27(\mathrm{~m}, 2 \mathrm{H}), 4.56(\mathrm{~m}, 1 \mathrm{H}), 3.24(\mathrm{~m}, 1 \mathrm{H}), 2.94(\mathrm{dd}, J$ $=17.6$ and $8.5 \mathrm{~Hz}, 1 \mathrm{H}), 2.79(\mathrm{dd}, J=17.6$ and $11.1 \mathrm{~Hz}, 1 \mathrm{H}), 1.42(\mathrm{~d}, J=6.2 \mathrm{~Hz}, 3 \mathrm{H}) ;{ }^{13} \mathrm{C}$ NMR (100 MHz) $\delta 175.4,138.2,129.0,127.7,127.1,83.0,49.5,37.3,19.1 ; \mathrm{MS}(\mathrm{m} / \mathrm{z}$, EI) $176\left(\mathrm{M}^{+}\right)$.<smiles>CC1OC(=O)C[C@@H]1Pc1ccccc1</smiles>

cis-Dihydro-5-methyl-4-phenyl-2(3H)-furanone (Table 4, entry 6). ${ }^{\mathbf{1 4}}{ }^{1} \mathrm{H}$ NMR (400 MHz) $\delta 7.37-7.29(\mathrm{~m}, 3 \mathrm{H}), 7.15-7.13(\mathrm{~m}, 2 \mathrm{H}), 4.93(\mathrm{~m}, 1 \mathrm{H}), 3.76(\mathrm{~m}, 1 \mathrm{H}), 2.95(\mathrm{dd}, J$ $=17.6$ and $9.0 \mathrm{~Hz}, 1 \mathrm{H}), 2.81(\mathrm{dd}, J=17.6$ and $6.1 \mathrm{~Hz}, 1 \mathrm{H}), 1.00(\mathrm{~d}, J=6.5 \mathrm{~Hz}, 3 \mathrm{H}) ;{ }^{13} \mathrm{C}$ NMR $(100 \mathrm{MHz}) \delta 176.6,137.5,128.6,127.6,127.4,79.8,44.6,34.7,16.5 ; \mathrm{MS}(\mathrm{m} / \mathrm{z}$, EI) $176\left(\mathrm{M}^{+}\right)$.

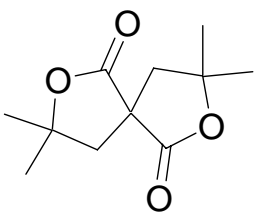

3,3,8,8-Tetramethyl-2,7-dioxaspiro[4.4]nonane-1,6-dione (Table 4, entry 7). ${ }^{15}{ }^{1} \mathrm{H}$ NMR (400 MHz) $\delta 2.80(\mathrm{~d}, J=13.4 \mathrm{~Hz}, 2 \mathrm{H}), 2.17(\mathrm{~d}, J=13.4 \mathrm{~Hz}, 2 \mathrm{H}), 1.59(\mathrm{~s}, 6 \mathrm{H})$, 1.45 (s, 6H); ${ }^{13} \mathrm{C}$ NMR (100 MHz) $\delta 174.1,83.5,55.0,47.7,29.3,28.4 ; \mathrm{MS}(\mathrm{m} / \mathrm{z}, \mathrm{EI}) 212$ $\left(\mathrm{M}^{+}\right)$.

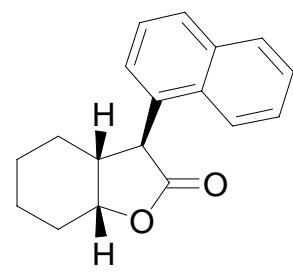

exo-cis-Hexahydro-endo-3-naphthyl-2(3H)-benzofuranone (Table 4, entry 8). ${ }^{1} \mathrm{H}$ NMR $(400 \mathrm{MHz}) \delta 7.94-7.88(\mathrm{~m}, 2 \mathrm{H}), 7.82(\mathrm{~d}, J=8.2 \mathrm{~Hz}, 1 \mathrm{H}), 7.69(\mathrm{~d}, J=7.1 \mathrm{~Hz}$, 1H), $7.57-7.48(\mathrm{~m}, 3 \mathrm{H}), 4.77(\mathrm{q}, J=4.0 \mathrm{~Hz}, 1 \mathrm{H}), 4.76(\mathrm{~d}, J=6.1 \mathrm{~Hz}, 1 \mathrm{H}), 2.81(\mathrm{~m}$, $1 \mathrm{H}), 2.35(\mathrm{~m}, 1 \mathrm{H}), 1.67(\mathrm{~m}, 1 \mathrm{H}), 1.57(\mathrm{~m}, 2 \mathrm{H}), 1.45(\mathrm{~m}, 1 \mathrm{H}), 1.04(\mathrm{~m}, 2 \mathrm{H}), 0.79(\mathrm{~m}, 1 \mathrm{H})$; 
${ }^{13} \mathrm{C}$ NMR $(100 \mathrm{MHz}) \delta 176.8,133.6,131.8,129.1,128.5,128.0,127.3,126.3,125.6$, 125.2, 122.0, 77.0, 50.0, 40.1, 27.6, 24.1, 23.1, 19.7; MS (m/z, EI) $266\left(\mathrm{M}^{+}\right)$.

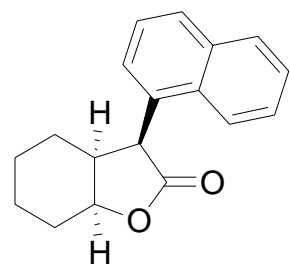

endo-cis-Hexahydro-exo-3-naphthyl-2(3H)-benzofuranone (Table 4, entry 8). ${ }^{1} \mathrm{H}$ NMR $(500 \mathrm{MHz}) \delta 7.88(\mathrm{dd}, J=7.7$ and $1.1 \mathrm{~Hz}, 1 \mathrm{H}), 7.82(\mathrm{~d}, J=7.9 \mathrm{~Hz}, 1 \mathrm{H}), 7.80(\mathrm{~d}, J$ $=7.9 \mathrm{~Hz}, 1 \mathrm{H}), 7.57-7.50(\mathrm{~m}, 2 \mathrm{H}), 7.42(\mathrm{dd}, J=7.9$ and $7.9 \mathrm{~Hz}, 1 \mathrm{H}), 7.28(\mathrm{~d}, J=7.1 \mathrm{~Hz}$, $1 \mathrm{H}), 4.65(\mathrm{q}, J=4.6 \mathrm{~Hz}, 1 \mathrm{H}), 4.28(\mathrm{~d}, J=4.1 \mathrm{~Hz}, 1 \mathrm{H}), 2.52(\mathrm{~m}, 1 \mathrm{H}), 2.10-2.01(\mathrm{~m}$, $2 \mathrm{H}), 1.74-1.51(\mathrm{~m}, 5 \mathrm{H}), 1.36-1.30(\mathrm{~m}, 1 \mathrm{H}) ;{ }^{13} \mathrm{C}$ NMR $(125 \mathrm{MHz}) \delta 178.3,134.1$, $131.9,131.4,129.1,128.3,126.5,125.9,125.3,124.9,122.8,77.3,51.8,43.2$, 27.8, 26.9, 22.6, 20.2; MS (m/z, EI) $266\left(\mathrm{M}^{+}\right)$.

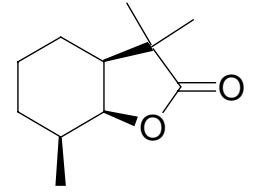

cis,cis-Hexahydro-3,3,7-trimethyl-2(3H)-benzofuranone (10). ${ }^{1} \mathrm{H}$ NMR $(500 \mathrm{MHz}) \delta$ $4.48(\mathrm{dd}, J=4.0$ and $3.5 \mathrm{~Hz}, 1 \mathrm{H}), 1.91(\mathrm{ddd}, J=12.0$ and 5.5 and $4.0 \mathrm{~Hz}, 1 \mathrm{H}), 1.72(\mathrm{~m}$, $1 \mathrm{H}), 1.65(\mathrm{~m}, 2 \mathrm{H}), 1.48(\mathrm{~m}, 1 \mathrm{H}), 1.27(\mathrm{~s}, 3 \mathrm{H}), 1.19-1.08(\mathrm{~m}, 2 \mathrm{H}), 1.13(\mathrm{~s}, 3 \mathrm{H}), 1.11(\mathrm{~d}$, $J=7.0 \mathrm{~Hz}, 3 \mathrm{H}), 1.06-0.99(\mathrm{~m}, 1 \mathrm{H}) ;{ }^{13} \mathrm{C} \mathrm{NMR}(125 \mathrm{MHz}) \delta 182.2,79.5,45.8,45.4$, 34.0, 27.8, 24.3, 23.8, 22.9, 19.1, 18.3; MS (m/z, EI) $182\left(\mathrm{M}^{+}\right)$.

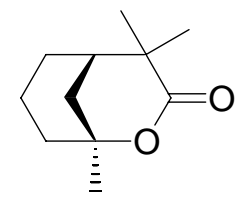

1,4,4-Trimethyl-2-oxabicyclo[3.3.1]nonan-3-one (11). ${ }^{1} \mathrm{H}$ NMR $(500 \mathrm{MHz}) \delta 2.13$ (dq, $J=14.0$ and $2.5 \mathrm{~Hz}, 1 \mathrm{H}), 1.91-1.87(\mathrm{~m}, 2 \mathrm{H}), 1.76(\mathrm{~m}, 1 \mathrm{H}), 1.61-1.58(\mathrm{~m}, 2 \mathrm{H}), 1.44-$ $1.35(\mathrm{~m}, 3 \mathrm{H}), 1.33(\mathrm{~s}, 3 \mathrm{H}), 1.32(\mathrm{~s}, 3 \mathrm{H}), 1.26(\mathrm{~s}, 3 \mathrm{H}) ;{ }^{13} \mathrm{C} \mathrm{NMR}(125 \mathrm{MHz}) \delta 179.1,81.3$, 39.4, 38.2, 37.5, 34.9, 29.6, 29.0, 26.9, 23.7, 17.6; MS (m/z, EI) $182\left(\mathrm{M}^{+}\right)$.

\section{Reference}

1. Qian, H.; Han, X.; Widenhoefer, R. A. J. Am. Chem. Soc. 2004, 126, 9536-9537.

2. Dussalt, P. H.; Zope, U. R. J. Org. Chem. 1995, 60, 8218-8222.

3. Harnden, M. P.; Jarvest, R. L. J. Chem. Soc. Perkin Trans. I 1991, 2073-2079.

4. Clarke, P. A. Tetrahedron Lett. 2002, 43, 4761-4763.

5. Breton, G. W.; Shugart, . H.; Hughey, C. A.; Conrad, B. P.; Perala, S. M. Molecules [online computer file], 2001, 6, 655-662. 
6. Fujita, T.; Watanabe, S.; Suga, K.; Nakayama, H. Synthesis 1979, 310-311.

7. Li, W.; Hanau, C. E.; dAvignon, A.; Moeller, K. D. J. Org. Chem. 1995, 60, 8155-8170.

8. Bien, S.; Ovadia, D. J. Chem. Soc. Perkin Trans. I 1974, 333-336.

9. Horvat, S.; Karallas, P.; White, J. M. J. Chem. Soc. Perkin Trans. II 1998, 21512154.

10. Tiecco, M.; Tingoli, M.; Testaferri, L.; Bartoli, D. Synth. Commun. 1989, 19, 2817-2824.

11. Souma, Y.; Iyoda, J.; Sano, H. Bull. Chem. Soc. Jpn. 1976, 49, 3291-3295.

12. Jeanne-Carlier, R.; Bourelle-Wargnier, F. Tetrahedron Lett. 1975, 16, 1841-1842.

13. Reissig, H.-U.; Angert, H. J. Org. Chem. 1993, 58, 6280-6285.

14. Brown, E.; Deroye, C.; Touet, J. Tetrahedron: Asymmetry 1998, 9, 1605-1614.

15. Fristad, W. E.; Hershberger, S. S. J. Org. Chem. 1985, 50, 1026-1031. 
Figure S1 Olefin 1 in $\mathrm{CD}_{2} \mathrm{Cl}_{2}$ at $298 \mathrm{~K}$.

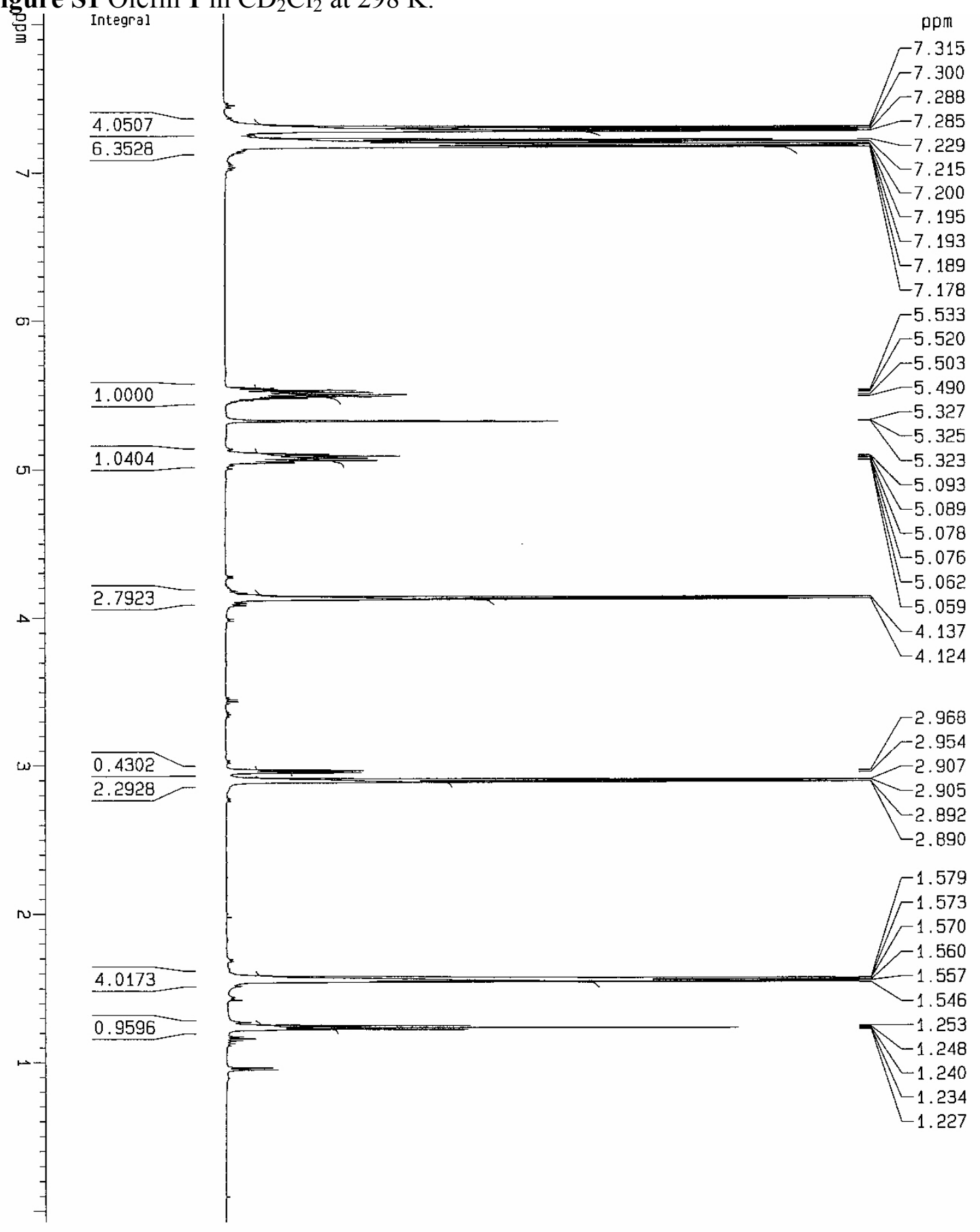


Figure S2 Olefin 1 with $20 \mathrm{~mol} \%$ of silver triflate in $\mathrm{CD}_{2} \mathrm{Cl}_{2}$ at $298 \mathrm{~K}$

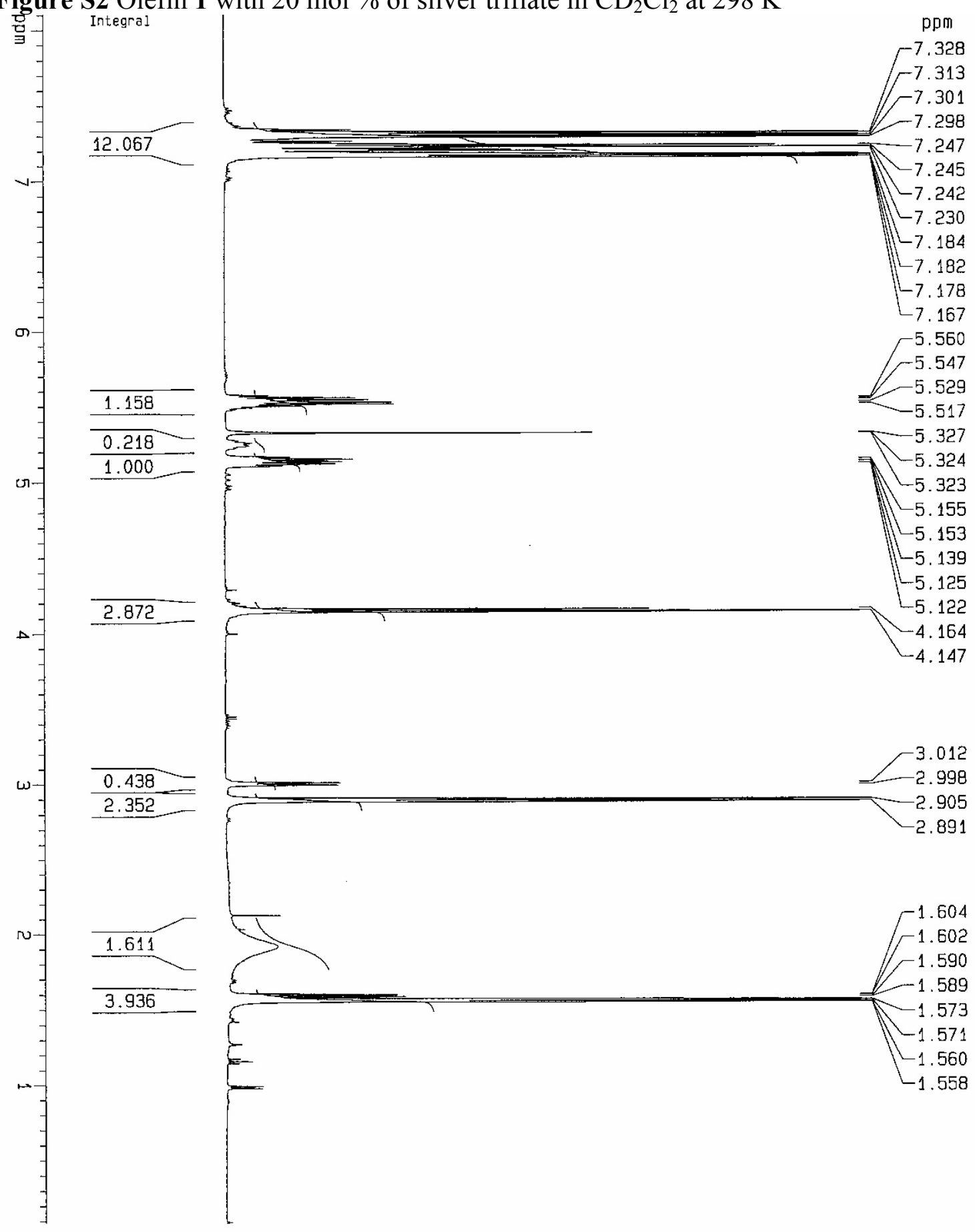


Figure S3 Olefin 1 with $50 \mathrm{~mol} \%$ of silver triflate in $\mathrm{CD}_{2} \mathrm{Cl}_{2}$ at $298 \mathrm{~K}$.

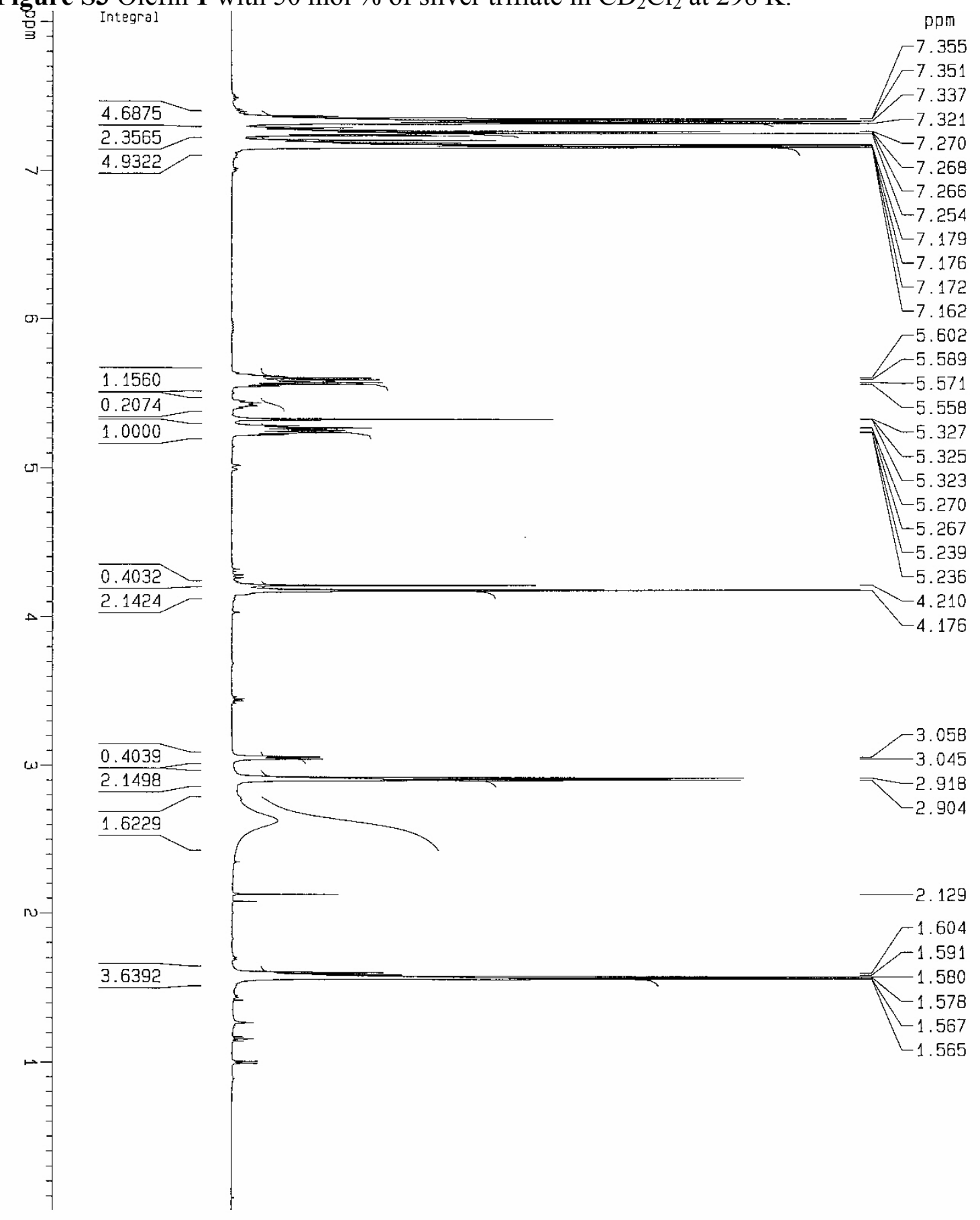


Figure S4 Olefin 1 with an equimolar of silver triflate in $\mathrm{CD}_{2} \mathrm{Cl}_{2}$ at $298 \mathrm{~K}$.

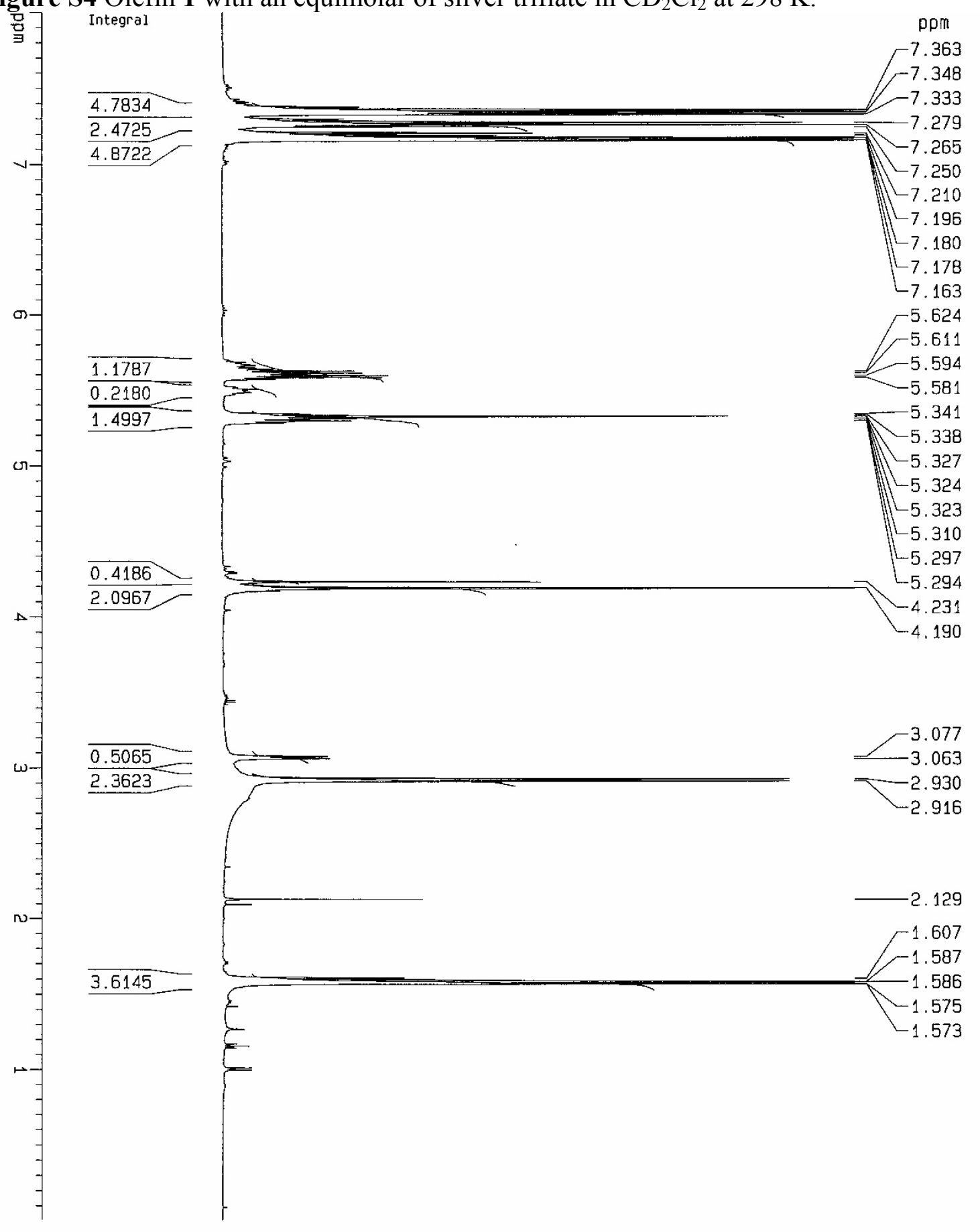


Figure S5 Olefin 1 in 1,2-dichloroethane- $\mathrm{d}_{4}$ at $353 \mathrm{~K}$.

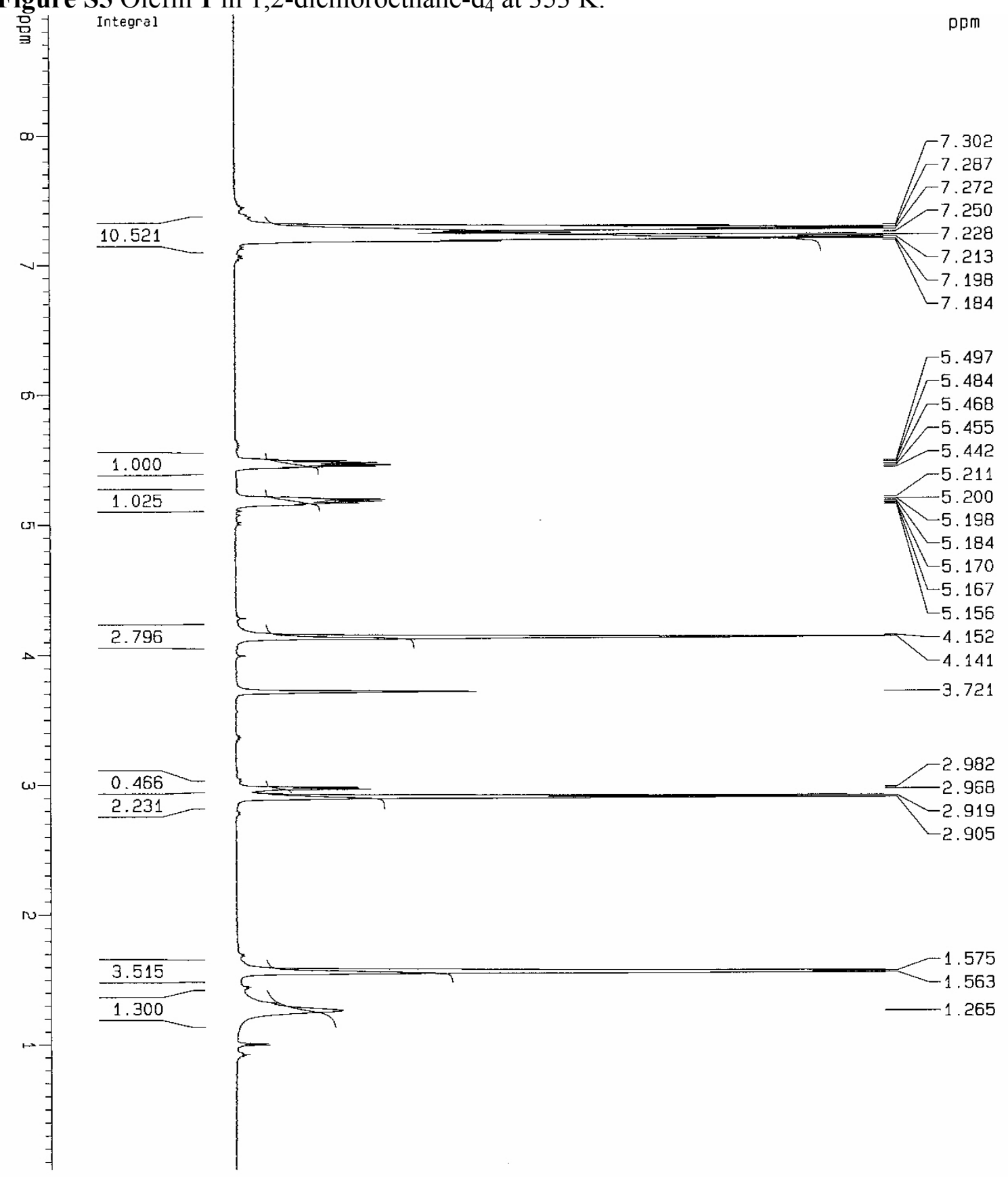


Figure S6 Olefin 1 with $50 \mathrm{~mol} \%$ Silver triflate in 1,2-dichloroethane-d $\mathrm{d}_{4}$ at $298 \mathrm{~K}$.

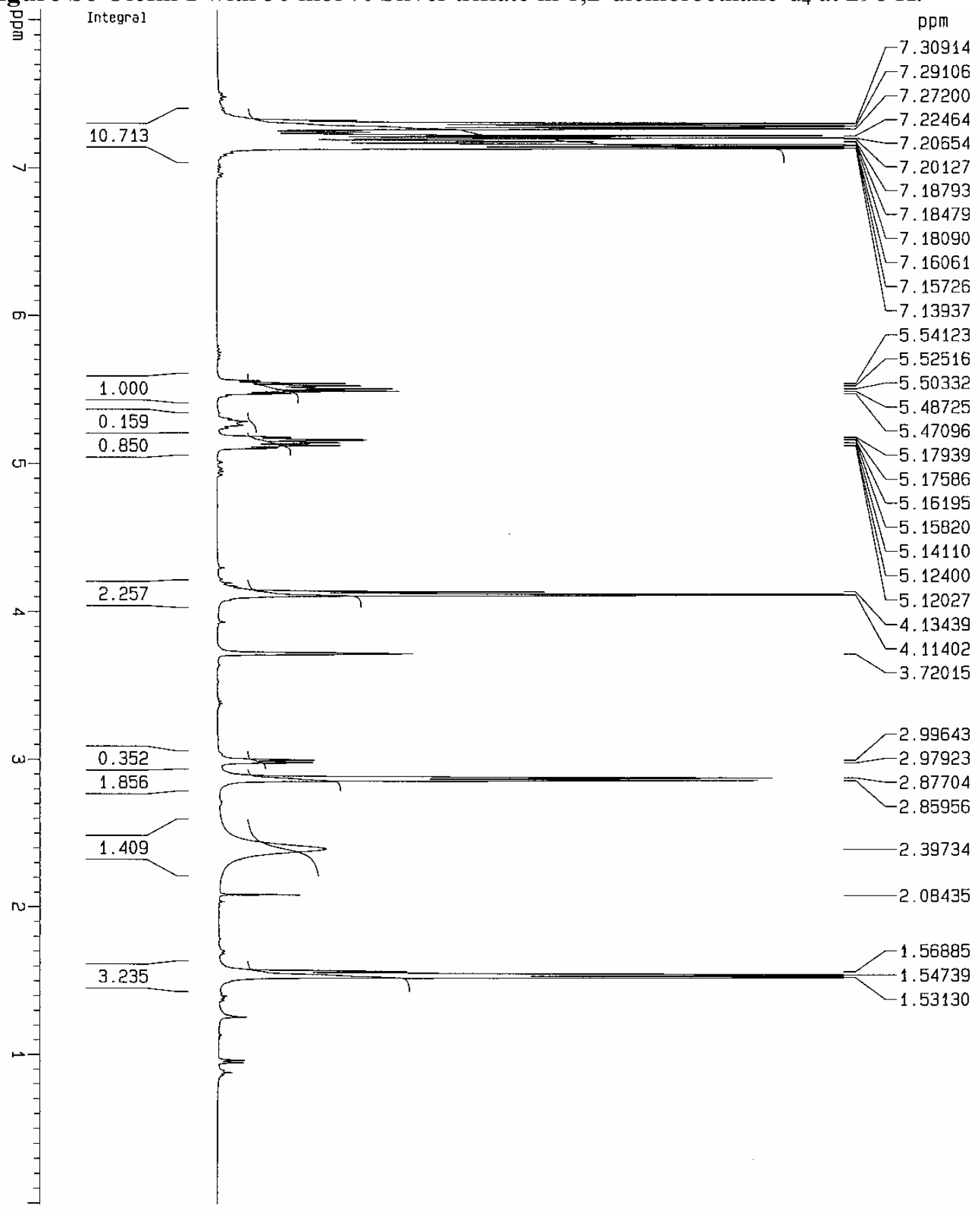


Figure S7 Olefin 1 with 50 mol \% Silver triflate in 1,2-dichloroethane-d $\mathrm{d}_{4}$ at $353 \mathrm{~K}$.

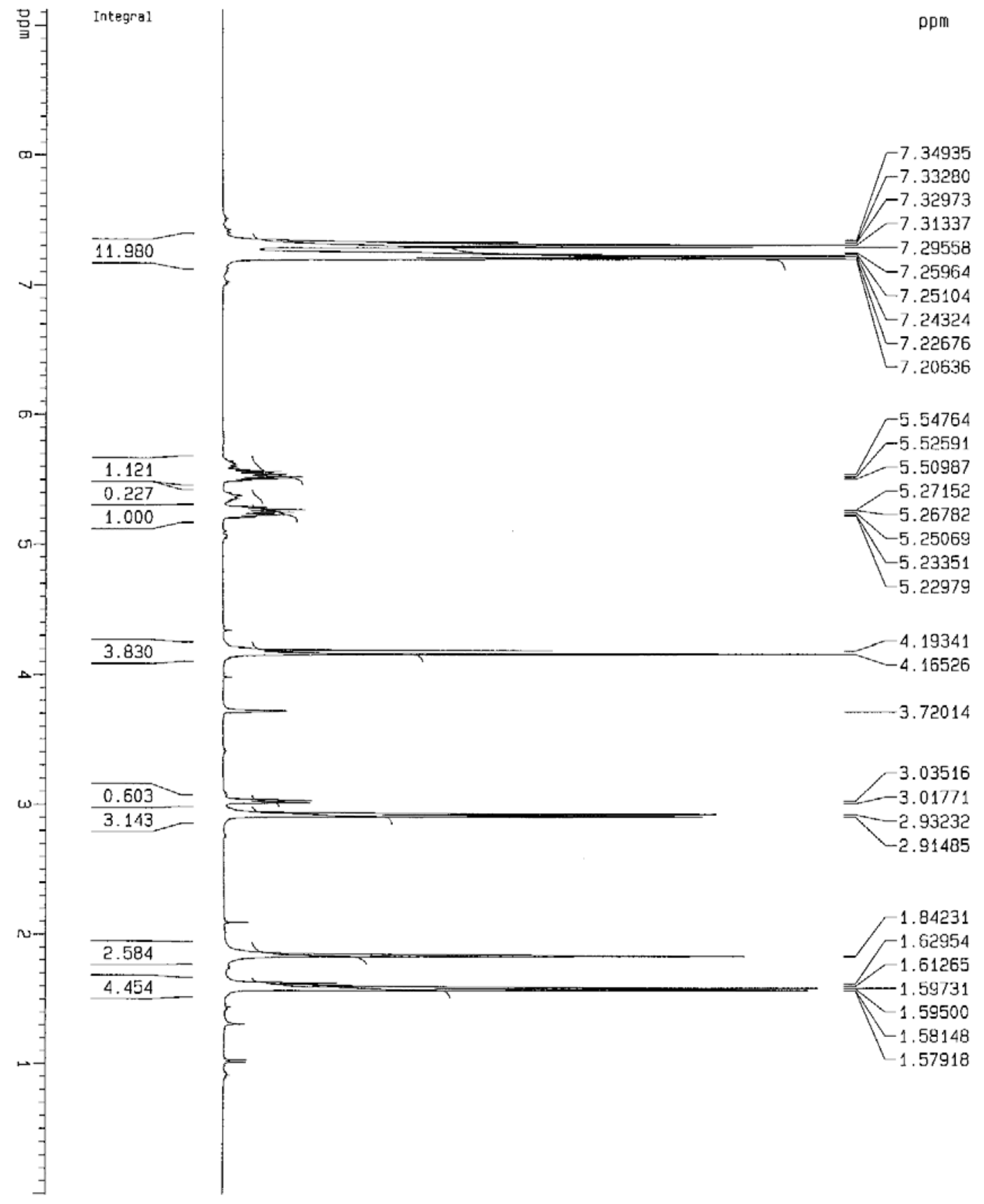


Figure S8 Olefin 1 with $50 \mathrm{~mol} \%$ Silver triflate in 1,2-dichloroethane- $\mathrm{d}_{4}$ at $353 \mathrm{~K}$ for 1.5 h.

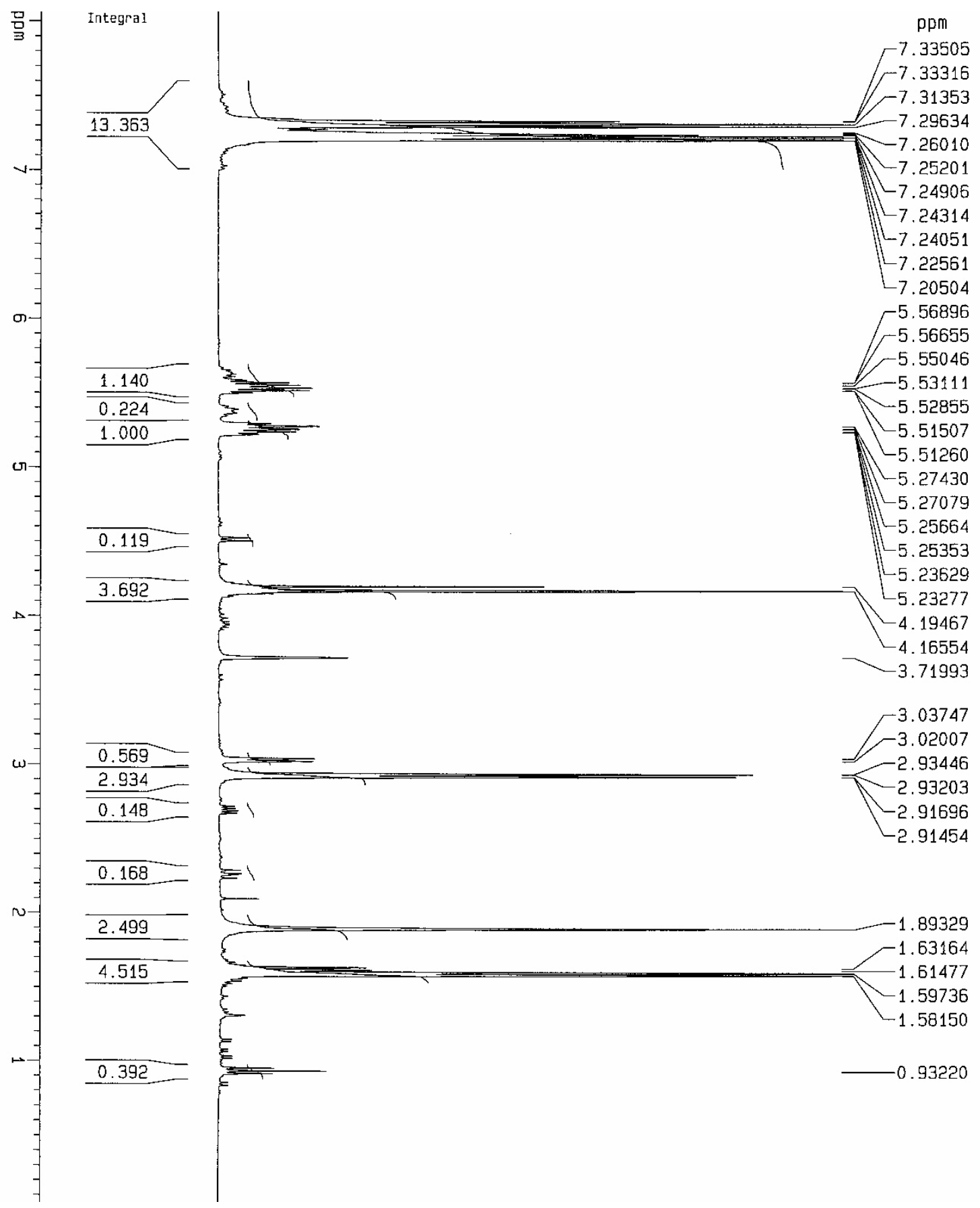


Figure S9 Olefin 1 with 50 mol \% Silver triflate in 1,2-dichloroethane- $\mathrm{d}_{4}$ at $353 \mathrm{~K}$ for 2.5 h.

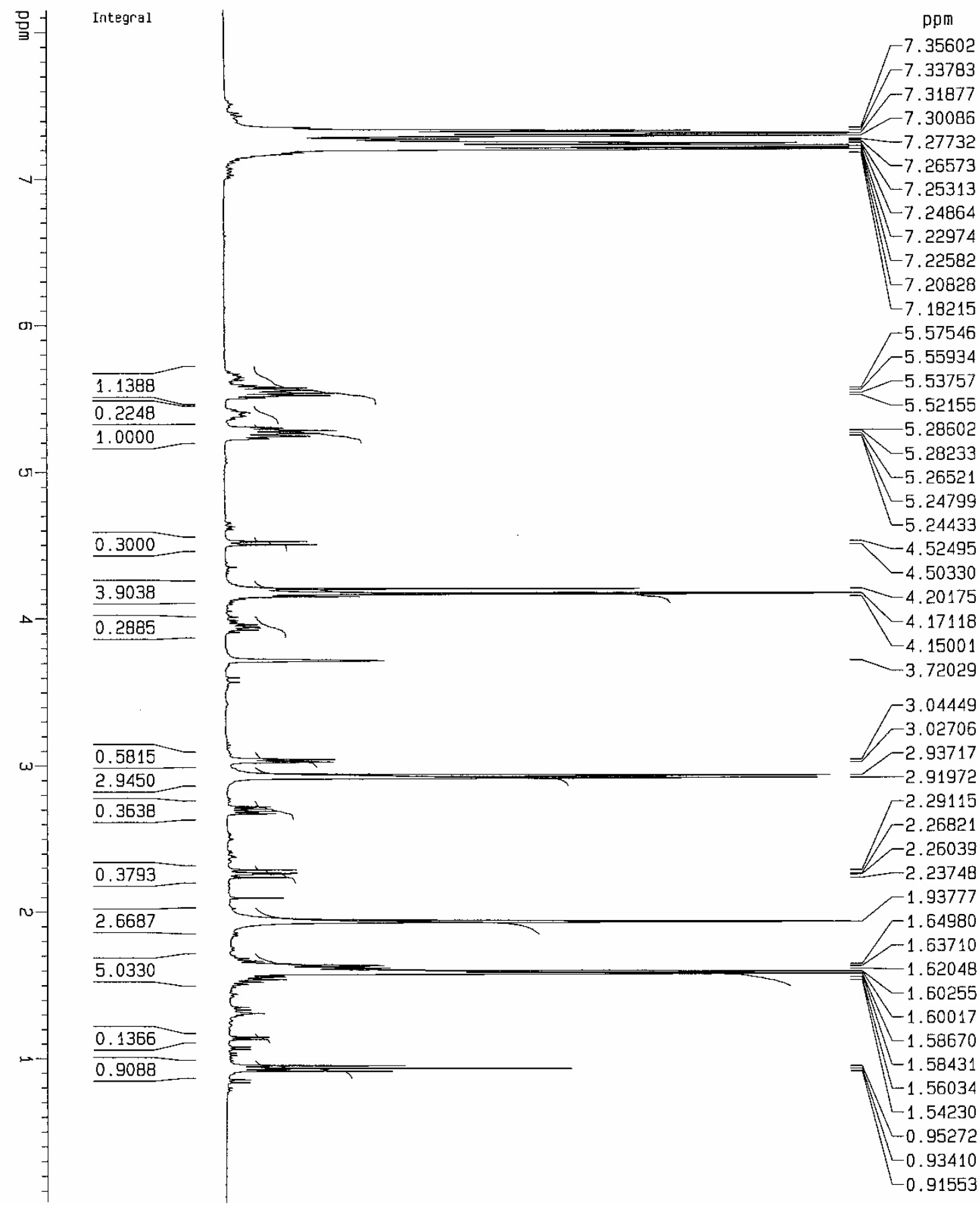


Figure S10 Olefin 1 with $50 \mathrm{~mol} \%$ Silver triflate in 1,2-dichloroethane- $\mathrm{d}_{4}$ at $353 \mathrm{~K}$ for $12 \mathrm{~h}$.

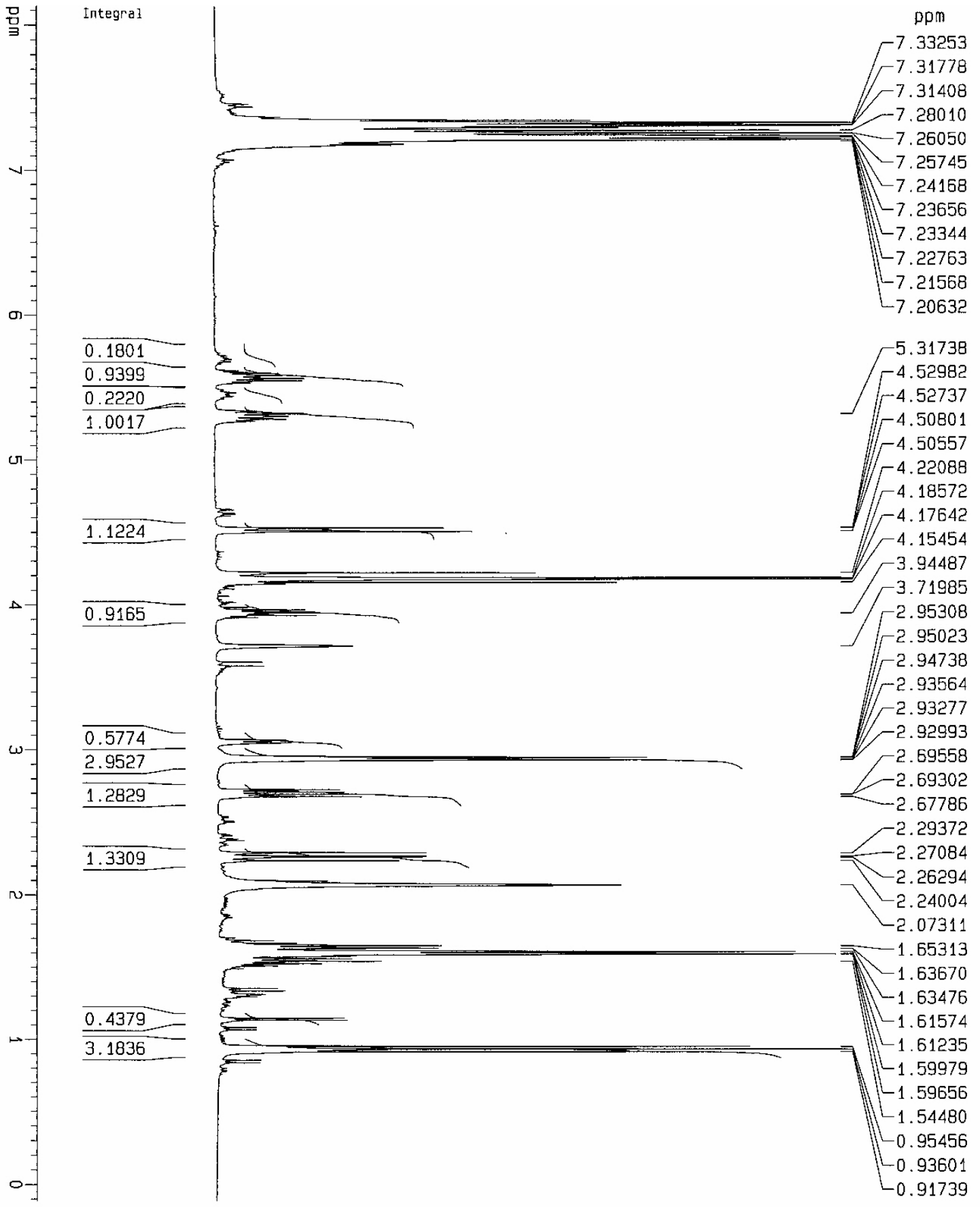


Figure S11 Excess amount of silver triflate was added to the reaction mixture at $353 \mathrm{~K}$.

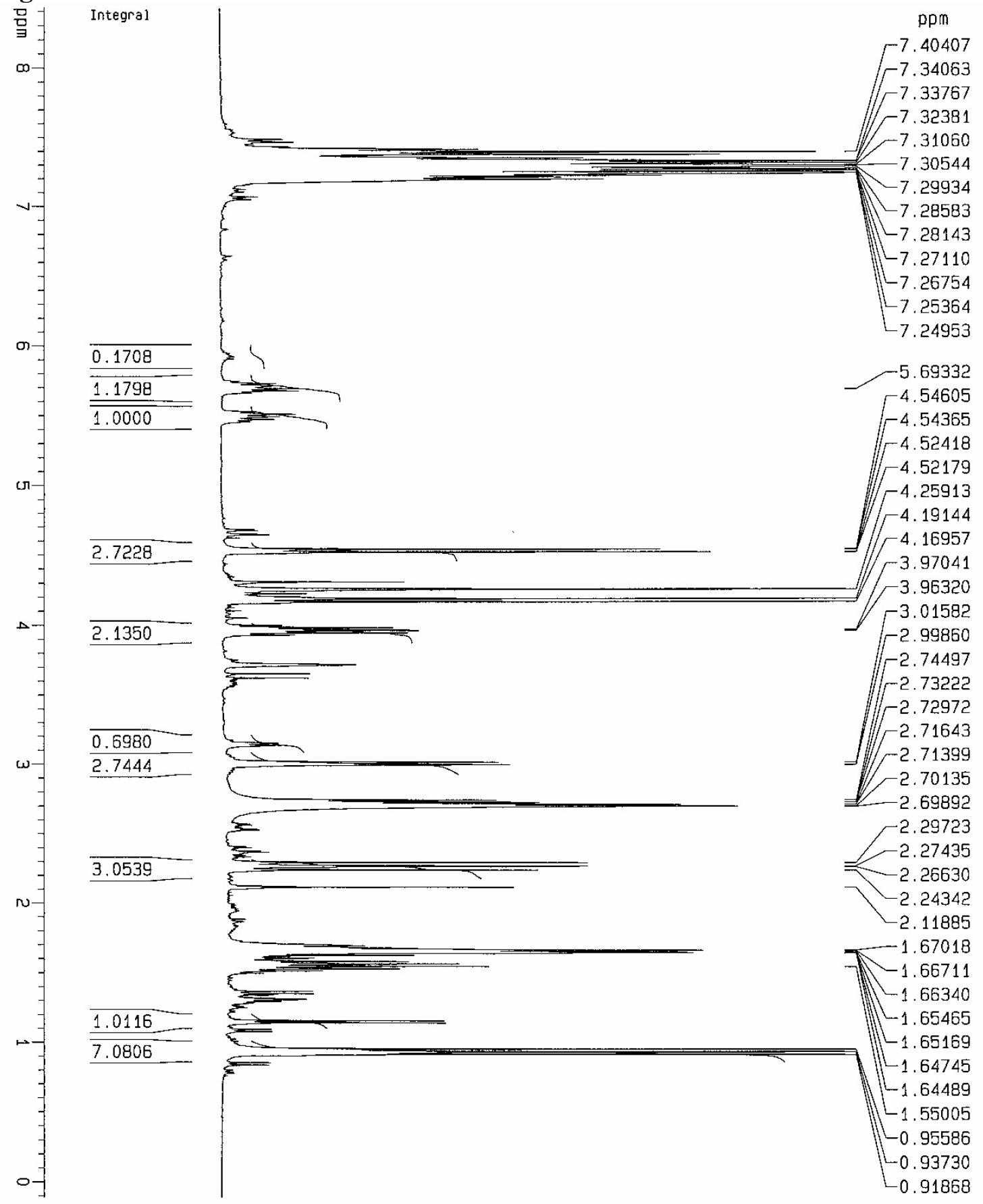


Figure S12 Excess amount of silver triflate was added to the reaction mixture at $353 \mathrm{~K}$ after $1.5 \mathrm{~h}$.

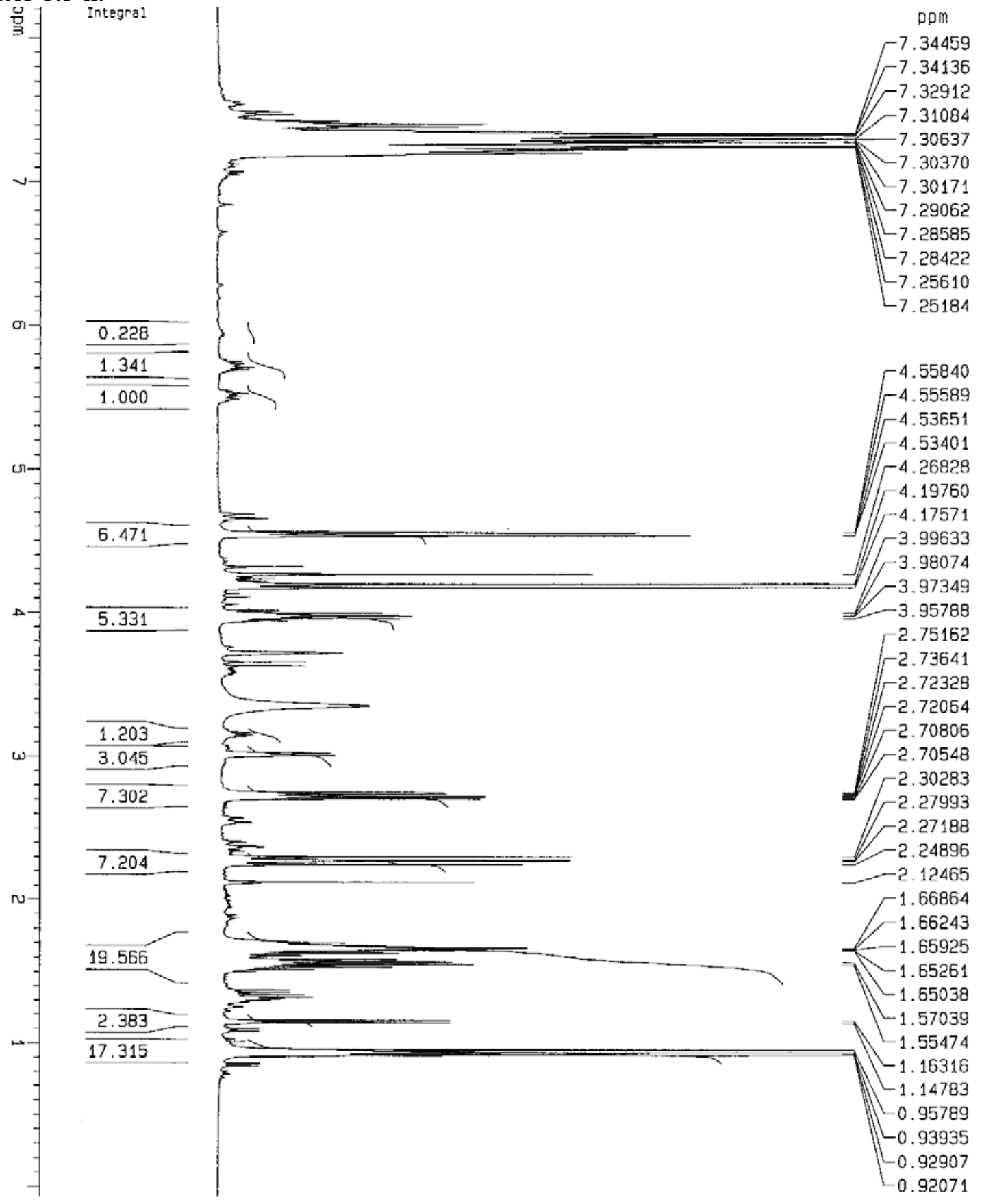


Figure S13 Excess amount of silver triflate was added to the reaction mixture at $353 \mathrm{~K}$ after $3.5 \mathrm{~h}$. Reaction complete.

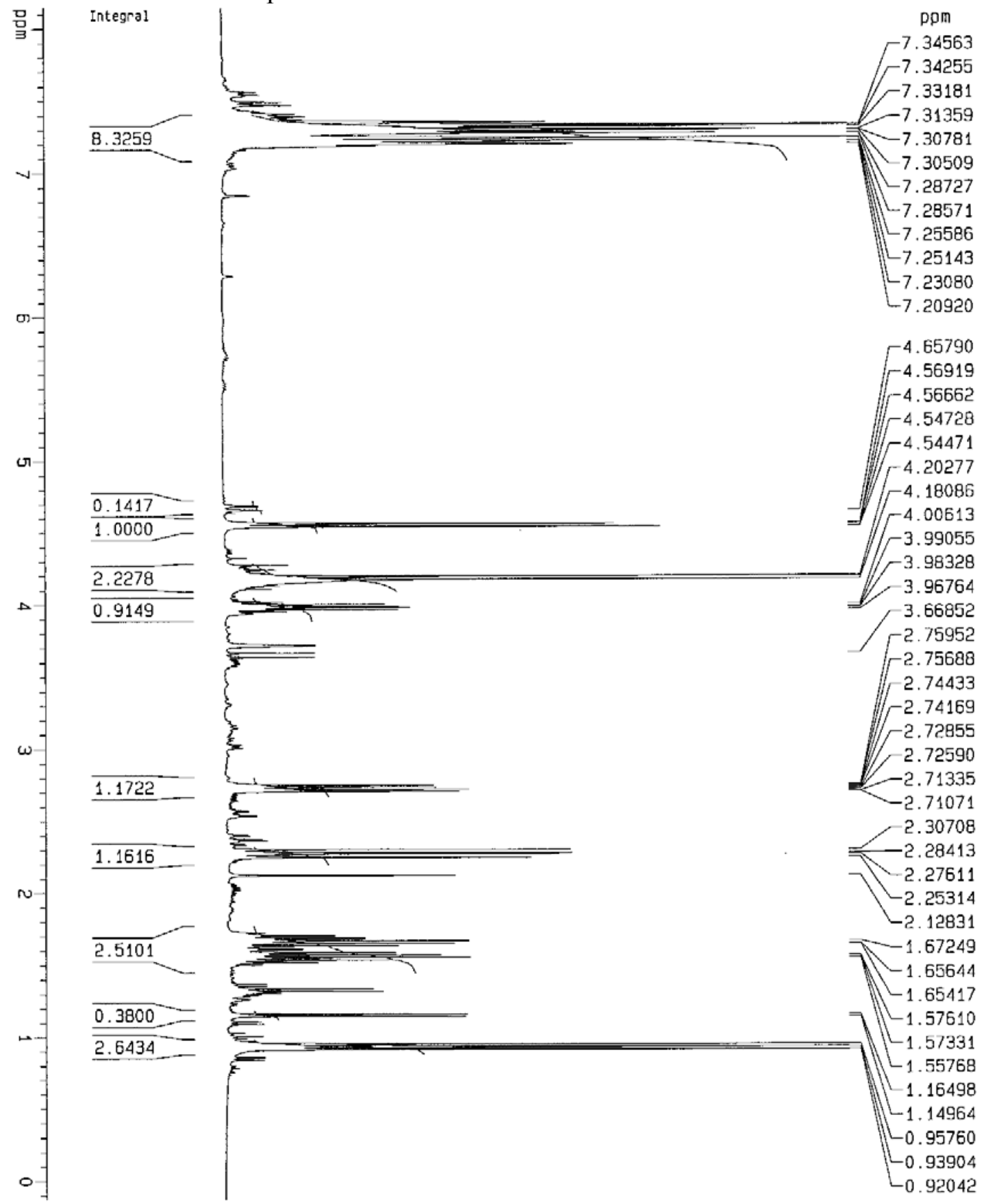

